NBER WORKING PAPER SERIES

\title{
THE DEMAND FOR COORDINATION
}

\author{
Wouter Dessein \\ Tano Santos \\ Working Paper 10056 \\ http://www.nber.org/papers/w10056 \\ NATIONAL BUREAU OF ECONOMIC RESEARCH \\ 1050 Massachusetts Avenue \\ Cambridge, MA 02138 \\ October 2003
}

We thank Gary Becker, Mathias Dewatripont, Jonathan Levin, Kevin Murphy, Canice Prendergast, and John Roberts for their comments. We also thank seminar participants at the Department of Economics and the Graduate School of Business of the University of Chicago, the Stanford Strategic Management Conference, the Wallace Summer Conference at Rochester, and the Stanford Institute of Theoretical Economics (SITE). A previous version of this paper was circulated under the title "Adaptive Organizations." All errors remain our own. The views expressed herein are those of the authors and not necessarily those of the National Bureau of Economic Research.

(C2003 by Wouter Dessein and Tano Santos. All rights reserved. Short sections of text, not to exceed two paragraphs, may be quoted without explicit permission provided that full credit, including (C) notice, is given to the source. 
The Demand for Coordination

Wouter Dessein and Tano Santos

NBER Working Paper No. 10056

October 2003

JEL No. D2, D8, J2, L2

\title{
ABSTRACT
}

This paper endogenizes coordination problems in organizations by allowing for both ex ante coordination of activities, using rules and task guidelines, and ex post coordination, using communication and broad job assignments. It shows that:

(i) Task specialization and the division of labor is mainly limited by employee discretion, rather than by the importance of coordination. In particular, specialization is often non-monotonic in the importance of coordination.

(ii) Organizations exhibit increasing returns to ex post coordination. This rationalizes discrete 'shifts' in organizational design from very rigid and specialized task assignments, to very flexible organizations characterized by extensive task bundling, intensive horizontal communication and substantial employee discretion.

(iii) Broad task assignments and intensive horizontal communication are complementary. Hence, lower communication costs often result in less specialization.

\author{
Wouter Dessein \\ Graduate School of Business \\ University of Chicago \\ wouter.dessein@gsb.uchicago.edu \\ Tano Santos \\ Graduate School of Business \\ Columbia University \\ 3022 Broadway, Uris Hall 414 \\ New York, NY 10027 \\ and NBER \\ js1786@columbia.edu
}




\section{INTRODUCTION}

Since Adam Smith (1981), specialization and the division of labor has been at the center of the organization of production. Whereas most economists share Smith's view about the importance of specialization for productivity and economic growth, there is more disagreement as to what limits the division of labor. In particular, many economists have argued that the benefits of specialization are mainly limited by the need to coordinate these specialized activities (Becker and Murphy (1992), Bolton and Dewatripont (1992)), rather than by the 'extent of the market,' as proposed by Smith (1981). ${ }^{1}$

Independently of whether one thinks the key determinant of the degree of specialization is the extent of the market or the need to coordinate specialized tasks, neither of these two theories seem to be able to account for recent changes in organizational practices. In particular, a growing body of empirical and anecdotal evidence has documented a trend towards 'new workplace practices' involving less specialized job assignments, more team work, and more intensive communication. ${ }^{2}$ This transformation of the organization of production has occurred at the same time that globalization has opened up markets and improvements in information technology have made coordination easier, ${ }^{3}$ the two forces that are supposed to lead to more specialization, not less.

This paper departs from the literature by endogenizing the demand for coordination. We show that this endogeneity is essential in understanding the problem of organizational design from a theoretical point of view as well as in rationalizing the changes in workplace practices recently observed. The key idea underlying our arguments is that the demand for coordination depends on the discretion which employees receive to adapt their tasks to local circumstances. Concretely, we propose a model in which activities can be coordinated ex ante, by letting employees stick tightly to rules and task-guidelines, and ex post, using communication and broad

\footnotetext{
${ }^{1}$ Also the business literature has identified and paid considerable attention to this trade-off. Indeed, according to Rivkin and Siggelkow (2002) "the [qualitative management] literature is unified in what it perceives as the central challenge of organizational design: to divide the tasks of a firm into manageable, specialized jobs, yet coordinate the tasks so that the firm reaps the benefits of harmonious action." For an early reference on the statement of the problem see March and Simon (1958, pages 22-30.)

${ }^{2}$ See, for example Caroli (2001), OECD (1999), Osterman (1994), Brynjolfsson and Hitt (1997), Caroli and Van Reenen (2001), and Ichniowski, Kochan, Levine, and Strauss (1996). Consistent with this trend towards less specialized jobs, the biggest management fad of the 1990s, re-engeneering [Hammer and Champy (2001)] prescribes 'combining several jobs into one' and, thus 'putting back together again the work that Adam Smith and Henry Ford broke into tiny pieces' (p.70).

${ }^{3}$ Information technology improves coordination by lowering communication costs and by allowing a better and more flexible integration of different production processes.
} 
job assignments. Specialization and the division of labor is then not mainly limited by the importance of coordination, but instead by the level of employee discretion. Intuitively, organizations can always perfectly coordinate their activities ex ante by letting employees tightly stick to some pre-agreed course of action. Alternatively, employees may be given substantial discretion to tailor their tasks to the local environment. Only in the latter case, specialization and limited communication result in coordination problems. ${ }^{4}$

Taking into account the endogeneity of the demand for coordination has important consequences for organizational design. First, task specialization is non-monotonic in task interdependence. Indeed, an increase in the importance of coordination affects two margins. First, for a given level of employee discretion, it will become more important to improve ex post coordination by increasing task bundling and improving horizontal communication (Becker and Murphy (1992)). Second, it will become optimal to constrain employee discretion, reducing the demand for ex post coordination. Tasks therefore tend to be most broadly defined for intermediate levels of task interdependence. Indeed, as task interdependence increases further, organizations increasingly rely on ex ante coordination, where specialized employees tightly stick to pre-scribed task guidelines. Specialization and the division of labor are then increasing in the costs associated with the lack of coordination.

A second consequence of the endogeneity of the demand for coordination is that task specialization is non-monotonic in improvements in communication technology: While better or cheaper communication allows for a better coordination of specialized tasks (Bolton and Dewatripont (1992) and Garicano (2000)), organizations take advantage of improvements in communication technology to boost employee discretion in order to become more adaptive. This increases the demand for ex post coordination and broader task assignments. In particular, improvements in communication technology may transform very rigid and very specialized organizations into flexible organizations characterized by broad task assignments, intensive horizontal communication and substantial employee discretion.

A third and final consequence is that organizations naturally exhibit increasing returns to coordination, resulting in discrete organizational changes and complementarities between broad task assignments, intensive horizontal communication and substantial employee discre-

\footnotetext{
${ }^{4}$ There is a growing literature (for example, Aghion and Tirole (1997) and Dessein (2002)), which looks at employee discretion from a contractual perspective: who has decision rights for a particular action. Given that incentives play no role in our model, there is no need to specify such decision rights. In contrast, employee discretion is an equilibrium phenomenon: how much do workers adhere to ex ante task guidelines or, in contrast, tailor their actions to local circumstances.
} 
tion. Organizations can improve ex post coordination of actions through more task bundling or through more intensive communication (frequent meetings, team work). We show that this organizational problem is naturally convex: the better actions are coordinated, the larger are the incentives to further improve ex post coordination. Intuitively, as ex post coordination improves it is optimal for the organization to become more adaptive and increase employee discretion. Conversely, any increase in employee discretion increases the demand for a better ex post coordination. As a consequence, in the absence of off-setting increasing costs to ex post coordination, organizations are either extremely rigid and specialized, and fully rely on ex ante coordination, or they exhibit substantial employee discretion and rely on extensive ex post coordination. Even a small change in an exogenous parameter may then result in a discrete organizational change.

If we do see intermediate levels of specialization, more task-bundling and more intensive horizontal communication are complementary instruments of ex post coordination. Intuitively, the lower the degree of specialization, the better tasks are coordinated ex post and, hence, the higher the equilibrium level of employee discretion. This, in turn, increases the overall demand for coordination, making it optimal to also improve the quality of communication between unbundled tasks. It follows that there will be more task bundling if the organization can simultaneously improve communication between unbundled tasks. In addition, both instruments of coordination move up or down together in response to environmental changes, yielding a number of unambiguous comparative static predictions.

Related Literature. The theoretical literature studying organizational design originated with the theory of teams of Marschak and Radner (1972) and, building on this, Cremer (1980). ${ }^{5}$ Whereas this literature studies the coordination of tasks when specialization implies that information is necessarily disperse, the present paper is one of very few who endogenizes the division of labor which causes these coordination problems. Cremer, for example, studies the optimal grouping of technological interdependent production units, but takes the number of units which are bundled together as given. A notable exception is Geanokoplos and Milgrom (1991), who offer a partial characterization of the optimal level of task bundling in a setting a la Cremer. However, their model, in which managers have limited time to process and collect information about a number of units, has very different implication than ours. In contrast to our

\footnotetext{
${ }^{5}$ A strand of this literature is concerned with the optimal design of information processing organizations (e.g. Radner (1993), Van Zandt (1999), Bolton and Dewatripont (1994), and Vayanos (2003)). With the exception of Vayanos (2003), these papers focus on situations in which there exist no interdependencies among tasks and, hence, they are less related to the present paper.
} 
model, for example, more task uncertainty results in more specialization as collecting information is then more important. Another important exception is Garicano (2000), which studies vertical specialization in knowledge acquisition, that is, what range of problems is solved by production workers and what range of problems is solved by management. Unlike the present paper and Cremer (1980), however, there is no need to coordinate the tasks of production workers or subunits, as there are no interdependencies between problems. As in Geanokoplos and Milgrom (1991), Garicano's main focus is on the characteristics of the vertical hierarchy. Finally, the trade-off between specialization and coordination is also emphasized in Becker and Murphy (1992), though they do not consider the endogeneity of the demand for coordination. In addition, they do not model the sources of the coordination costs that specialization would bring, nor any form of communication within the parties or the role of management, so their model has limited organizational design implications. They emphasize instead the impact of growth in human capital on the extent of specialization. ${ }^{6}$

Mainly based inductive grounds, the business literature has extensively argued that elements of organizational design and structure are strongly complementary, that is they have to 'fit' with one another. This notion of 'strategic fit' was made concrete in the economics literature by Milgrom and Roberts $(1988,1990)$, using the mathematics of complementarity or 'supermodularity'. Our approach differs from Milgrom and Roberts in that we derive this complementarity in an explicit model of production, without making any assumptions about cross-derivatives. In addition, the main focus of Milgrom and Roberts is on elements of manufacturing strategy as opposed to organizational design. For example, Milgrom and Roberts (1990) focus on the complementarities between the choice of technology, capital investments, and operating systems. ${ }^{7}$ A paper which does explicitly analyze complementarities between elements of organizational design is Holmstrom and Milgrom (1994). Their focus, however, is on the complementarities between worker discretion, high-performance incentives and worker ownership of assets.

Outline. The paper proceeds as follows. Section II introduces the model and Section III analyzes the behavior and performance of a given organizational structure. Section IV then discuss the implications for organizational design.

\footnotetext{
${ }^{6}$ Lindbeck and Snower (2000) is a more recent addition to this literature.

${ }^{7}$ In an overview paper, Milgrom and Roberts (1995) briefly discuss how some elements of human resource management policies are related to this modern manufacturing strategy, including horizontal communication and worker autonomy. They simply outline the assumptions on the cross-derivates of the reduced form profit function which are sufficient and necessary to guarantee the complementarity, however, without motivating these assumptions.
} 
In Section V, we offer several extensions of our framework. We first investigate the role of management in the organization of production. Management in our set up updates the guidelines and rules followed by workers. We show that a large managerial force and extensive vertical coordination are complementary to extensive task specialization and limited horizontal communication. To the best of our best knowledge, this is the first attempt to simultaneously analyze vertical and horizontal coordination. Aoki (1985), building on Cremer (1980), separately analyzes the efficiency of vertical and horizontal coordination of interdependent tasks and relates these to U.S. and Japanese work-practice. Unlike the present paper, however, his comparison of these two coordination modes yields no insight as to whether the latter are complements or substitutes, nor does he endogenize the optimal level of task bundling, and the extent of specialization.

We then extend the model to account for the interaction between organizational design, investments in IT and the demand for skills. We model skill as the ability to observe and process information that is relevant for task execution. We show that the demand for skills is complementary with broader task assignments and the quality of communication. The same is true for improvements and investments in information technology, such as data-bases and expert systems, which increase the ability of agents to adapt to local circumstances.

Finally, we explore alternative modeling choices regarding the nature of communication and find our results robust to these alternative choices.

Section VI relates our results to the mounting evidence on new organizational practices. Section VII concludes.

\section{THE MODEL}

In this section, we present a new team theoretic model of production, in which workers take actions after observing some local information and after having communicated with other workers involved in production. Organizational design determines the effectiveness of these actions through its impact on the information structure and productive efficiency of workers.

\section{II.A Adaptation and Coordination}

Production, in our model, requires the combination of $n$ tasks, where the profits of the organization depends on (i) how well each task is adapted to the organizational environment and (ii) how well each task is coordinated with the other tasks.

Adaptation.- Task $i$ consists of undertaking a primary action, $a^{i i}$, whose effectiveness depends on how well it is adapted to the local environment. Thus, adaptation calls for the use of 
local information, which exclusively pertains to a particular task and can only be observed by the worker assigned to it. This local information, a random variable $\theta^{i}$ with mean $\widehat{\theta}^{i}$ and a common variance $\sigma_{\theta}^{2}$, determines the optimal primary action. In particular, to achieve perfect adaptation, the primary action $a^{i i}$ should be set equal to $\theta^{i}$. The realization of the local information is independent across tasks.

Coordination. - In addition, in order to ensure that task $i$ is coordinated with all tasks $j \neq i$, the employee in charge of task $i$ must perform a string of $n-1$ actions $\left\{a^{i 1}, a^{i 2}, \cdots, a^{i n}\right\}$ which are complementary to the primary actions of task $j \neq i$. In particular, to achieve perfect coordination between task $i$ and $j$, action $a^{i j}$ of task $i$ should be set equal to the primary action $a^{j j}$.

For instance, if the organization consists of two tasks, then profits are maximized by minimizing the distance between the following two matrices

$$
\begin{array}{cl}
\text { Task } 1 & \longmapsto \\
\text { Task } 2 & \longmapsto
\end{array}\left(\begin{array}{cc}
a^{11} & a^{12} \\
a^{21} & a^{22}
\end{array}\right) \quad \text { and } \quad\left(\begin{array}{cc}
\theta^{1} & a^{22} \\
a^{11} & \theta^{2}
\end{array}\right)
$$

where the diagonal elements pertain to the adaptation objective and the off-diagonal elements to the coordination objective.

To illustrate our modeling choices consider the design of products and complex systems, activities where the trade-offs between adaptability and coordination are very prevalent. For example, coordination problems are particularly daunting in the case of the development of software projects large enough to be beyond the capabilities of a single software engineer. It is for this reason that large codes are broken into several modules, each being assigned to a different team. For instance, in the development of proprietary operating system, one module may be focused on the process manager whereas another is responsible for the network access. Each module will have actions that are specific to its function, the primary actions in our framework. At the same time though, the modules will have to be combined in one coherent whole. Software design choices geared towards the harmonious combination of these modules are the complementary actions in our set up. Uncertainty in software development springs from its non routine nature. Indeed, most projects are specifically tailored to the customer's needs and each module is likely to encounter peculiarities that are unique to that project. These peculiarities have to be addressed as the code is being written by the different teams and it is here where the trade-off between adaptability to the specific needs of the client and coordination across teams arises. ${ }^{8}$

\footnotetext{
${ }^{8}$ For a description of the coordination problems in the construction of proprietary operating systems see
} 
A similar case, taken from Hammer and Champy (2001, page 48), considers product design at Kodak: 'In a parallel design process, all the parts are designed simultaneously and integrated at the end. But this method engenders its own problem: Usually, the subsystems will not fit together because, even though all the groups were working from the same basic camera design, changes -often improvements- occurred along the way but were not communicated to the other groups. Then when the camera is supposed to be ready to go to production, it's back to square one in design.'

More generally, the success of organizations depends on how well they can respond to particular market conditions (demand may be higher or lower than expected,) operational conditions (a worker may be ill, an unexpected delay may occur), and how efficiently it can customize its products or services to particular consumer characteristics or changing consumer needs. Typically, the above desire for adaptation will result in coordination problems in the presence of specialization.

\section{II.B Organizational Design: Task Specialization}

An important objective of organizational design is to partition the totality of tasks into smaller jobs and assign them to specific individuals or groups. For conciseness, we will think of the organizational problem as the assignment of jobs to workers. The analysis of the assignment of jobs to subunits under the direction of a subunit manager, is formally identical.

Each task is assigned to exactly one employee, but an employee may have several tasks assigned to him. We denote by $\mathcal{T}(i)$ the set of tasks bundled with task $i$. To simplify the analysis we restrict the organization of production to be symmetric, that is, all workers inside the organization have an identical number $t$ of tasks assigned to them. That is, we exclusively consider organizations where $t \in \mathcal{J}=\left\{t \in \mathbb{N}\right.$ such that $\left.\frac{n}{t} \in \mathbb{N}\right\}$.

Task variety is costly in the usual specialization sense: as in Adam Smith's Pin Factory, the larger the number of tasks assigned to an employee, the lower his degree of specialization and, as a consequence, the lower his productivity. ${ }^{9}$ Concretely, the labor cost of carrying out task $i$, denoted by $h(t, \alpha)$, is increasing in the level of task bundling $t$, where the parameter $\alpha$ governs the returns to specialization. For instance, if a worker performs a broad collection of tasks it may take him longer to complete them, which is costly for the organization. Alter-

Crowston (1997). The literature devoted to coordination problems in software engineering is large. For example, see Zmud (1980), Curtis, Crasner, and Iscoe (1988), Kraut and Streeter (1995), Faraj and Sproull (2000) and the references therein.

${ }^{9}$ Alternatively, we could assume that task variety reduces the worker's ability to observe or adapt to the local conditions, $\theta^{i}$. We investigate this case in Section VI. 
natively, having broadly defined jobs may require more training or hiring more skilled agents who command higher wages. Formally,

$$
\Delta(\alpha)=h(\bar{t}, \alpha)-h(\underline{t}, \alpha)>0 \quad \text { and } \quad \Delta_{\alpha}>0 \quad \text { for } \quad \underline{t}, \bar{t} \in \mathcal{J} \quad \text { and } \quad \bar{t}>\underline{t} .
$$

Having introduced our concept of task specialization it is useful to return now to our modeling choices concerning adaptation and coordination. Workers, as we will see shortly, can communicate only imperfectly. Hence, in the presence of task specialization, adaptation to local circumstances puts at risk the harmonious coordination of specialized tasks. This trade-off between coordination and adaptation, however, completely disappears if all tasks are assigned to one worker. Perfect adaptation and coordination can then be simultaneously achieved.

Another modeling approach would be to only have primary actions which must be adapted both to local circumstances and to the primary actions of other tasks. This approach, however, introduces a technological trade-off between adaptation and coordination, which is present even in the absence of any division of labor. In our model, this trade-off is purely organizational, arising exclusively out of design choices involving specialization and communication. Introducing technological constraints on adaptation and coordination, would make the analysis more complicated, but it would not alter the main insight of the paper.

\section{II.C Organizational Design: Communication}

To improve coordination between specialized tasks, workers can communicate the choice of their primary action to other workers prior to its actual implementation. Such communication, however, will often be imperfect. Some reasons are, for example, the limitations on employees' ability to hold unscheduled meetings and the lack of a shared language that facilitates the quick transmission of information. As a result, an employee may not understand what the particular choice of a primary action by another employee implies for the corresponding complementary action under his control.

In particular, if task $i$ and $j$ are assigned to different employees, then with a probability $1-p^{i j}$, the message concerning the primary action of task $i$ will be pure noise for the employee in charge of task $j$. In contrast, with a probability $p^{i j}$, the agent in charge of task $j$ perfectly understands what the choice of action $a^{i i}$ means for the optimal choice of the complementary action $a^{j i}$.

$p^{j i}$ is an organizational design variable and we refer to it as the effectiveness or quality of the communication channels between two non-bundled tasks $i$ and $j$. We assume that the cost 
of improving communication between tasks is given by $\delta g\left(p^{i j}\right)$ where $g_{p}>0, g_{p}(0)=0$, and $\lim _{p \rightarrow 1} g_{p}(p)=\infty$, and $\delta$ is a positive constant. One can interpret $\delta g\left(p^{i j}\right)$ as the opportunity cost to the organization of having workers engaged in regular meetings, conference calls and electronic mail conversations, designed to exchange information rather than in production itself. Similarly, the organization can improve communication channels by job-rotation, team-events or by hiring employees with knowledge or skills which span across job-boundaries. Given the symmetry of our model, we can restrict $p^{i j}=p$ for all $i, j$ with $j \notin \mathcal{T}(i)$ without any loss of generality.

We assume that communication occurs task-to-task: Even if task $j$ and task $k$ are both allocated to the same worker and he understands the implications of $a^{i i}$ for task $j$, this does not imply that he understands what it means for task $k$. Therefore, the worker in charge of task $i$ must communicate $a^{i i}$ twice to the one in charge of task $j$ and $k$, incurring $\delta g(p)$ twice. Given a level of task bundling $t$, the total cost of the organization's communication network thus equals

$$
n(n-t) \delta g(p)
$$

Task-to-task communication is a natural assumption when the way a particular task is carried out cannot be easily summarized and affects different tasks differently. Communication in this case focuses on the interface between two tasks. For example, in the case of product development at Kodak, improvements implemented by the shutter designers are likely to have different implications for the camera body designers and the film mechanism designers. For each task pair, the agents in charge must then discuss how to coordinate these two tasks, incurring the cost $\delta g(p)$ along the way. From a theoretical perspective, task-to-task communication has the appealing feature that the communication costs between two unbundled task $i$ and $j$ are independent of the degree of specialization.

An alternative assumption, task-to-person communication, might be more appropriate when tasks are sufficiently simple such that they can be comprehensively described in a nutshell. In this case, an employee who understands what the choice of primary action for task $i$ means for task $j$ is also likely to understand the implications for any other task $k \in \mathcal{T}(j)$. We explore the implications of task-to-person communication in Section VI and show that it introduces a mechanical complementarity between task-bundling and communication quality. In particular, the communication costs between two unbundled tasks $i$ and $j$ are then increasing in the degree of specialization. In contrast, in our model with task-to-task communication, the complementarity between $p$ and $t$ is uniquely due to the endogenous demand for coordination.

Finally we assume that an agent never knows whether his communication with other 
agents was successful or not. Thus, when deciding upon a primary action $a^{i i}$, he takes into account that with a probability $p$, the employee in charge of $a^{j i}$ will be influenced by his communication on $a^{i i}$. This greatly simplifies both the analysis and exposition, and it does not affect any of our results qualitatively.

\section{II.D The Production Function}

Define $\bar{a}^{i}=\left(a^{1 i}, a^{2 i}, \cdots, a^{n i}\right)$ as the vector consisting of the primary action of task $i$, $a^{i i}$, and the string of actions belonging to task $j \neq i$ which are complementary to this primary action $a^{i i}$. The organization's profits are then given by

$$
\pi\left(\bar{a}^{1}, \bar{a}^{2}, \ldots, \bar{a}^{n}, p, t \mid \theta^{1}, \theta^{2}, \ldots, \theta^{n}\right) \equiv-\sum_{i=1}^{n} C^{i}\left(\bar{a}^{i}, p, t \mid \theta^{i}\right)
$$

where

$$
C^{i}\left(\bar{a}^{i}, p, t \mid \theta^{i}\right) \equiv \phi\left(a^{i i}-\theta^{i}\right)^{2}+\sum_{j \neq i} \beta\left(a^{j i}-a^{i i}\right)^{2}+(n-t) \delta g(p)+h(t, \alpha)
$$

represents the production costs related to the primary action of task $i$.

As noted above, the effectiveness of the primary action $a^{i i}$ depends on how close it is set to the local local circumstances $\theta^{i}$, capturing the need for adaptation of task $i$ to the organizational environment. The effectiveness of the complementary actions $a^{j i}, j \neq i$, depends

on how close they are set to the choice of $a^{i i}$, capturing the need for coordination between the primary action of task $i$ and the other tasks. The parameters $\phi$ and $\beta$ determine the importance of adaptation and coordination respectively.

The labor costs of task $i$, denoted by $h(t, \alpha)$, are increasing in the level of task-bundling $t$. Finally, $(n-t) \delta g(p)$ represents the cost of building and maintaining the quality of the communication channels which inform the $(n-t)$ tasks which are not carried out by the employee in charge of task $i$ about the latter's primary action.

\section{II.E Timing}

The timing of our model goes as follows:

(i) Organizational design stage: The organization determines the number of task per agent, $t$ and the quality of the communication channels, $p$.

(ii) The local circumstances $\theta^{i}, i=1,2, . ., n$, are realized and observed by the employee in charge of task $i$.

(iii) Communication stage: Workers communicate their intended choice of primary actions to each other. With an independent probability $p$, these communications are successful. 
(iv) Action stage: For all $i=1,2, . ., n$, the employee in charge of task $i$ chooses actions $a^{i j}, j=1,2, \ldots, n$, in such a way as to maximize the objective function (2), subject to his information constraints.

\section{ORGANIZATIONAL ACTIONS AND PERFORMANCE}

Production requires the combination of $n$ tasks; each of these involves the choice of a primary action that needs to be adapted to the local circumstances, and $n-1$ complementary actions which must be coordinated with the $n-1$ primary actions of other tasks. We start by characterizing the choice of these actions as a function of a particular organizational design $(p, t)$ in Lemma 1 and then evaluate the costs associated with that particular organizational design in Lemma 2.

Lemma 1 There exists a unique equilibrium in which workers, faced with a vector of local circumstances $\left(\theta^{1}, \theta^{2}, \ldots, \theta^{n}\right)$, choose the following primary and complementary actions:

$$
a^{i i}=\widehat{\theta}^{i}+\left(\frac{\phi}{\phi+B}\right)\left(\theta^{i}-\widehat{\theta}^{i}\right)
$$

with

$$
B=\beta(n-t)(1-p)
$$

and

$$
a^{j i}=\left\{\begin{array}{lll}
a^{i i} & \text { when task } j \text { learns } & a^{i i} \\
\widehat{\theta}^{i} & \text { when task } j \text { does not learn } & a^{i i}
\end{array},\right.
$$

Expression (4), which gives the choice of the primary action, has two terms. The first one is the expected value of the local circumstances. The second term captures the need for adaptation to local information, $\theta^{i}$, limited by the term $\phi /(\phi+B)$. Henceforth, will refer to

$$
\sigma^{2}\left(a^{i i}\right) \equiv\left(\frac{\phi}{\phi+B}\right)^{2} \sigma_{\theta}^{2}
$$

as the equilibrium level of discretion exercised by employees. Thus, $\sigma^{2}\left(a^{i i}\right)$ characterizes how strictly employees adhere to an ex ante plan of action or, in contrast, tailor their actions to local circumstances.

Employee discretion is increasing in the importance of local adaptation, $\phi$, the variability of the local circumstances, $\sigma_{\theta}^{2}$, and is decreasing in the term $B=\beta(n-t)(1-p)$, which measures the limits to discretion and adaptation that result from the need to maintain some coordination with other tasks. 
As one might expect, $B$ is decreasing in $t$, the number of tasks per agent: a reduction in specialization results in primary actions that are more adaptive to the local information as now the worker can rely on a perfect coordination with the additional tasks under his control. Also, $B$ is decreasing in $p$ : Increasing the quality of the communication channels naturally improves coordination as now complementary actions are based on better information. This results in more adaptive primary actions because the employees can feel confident that these actions are more likely to be understood by other agents in the organization. Finally, $B$ is increasing in $\beta$, which captures the overall importance of maintaining coordination in the organization.

Substituting (4) and (6) in the profit function (2) and taking expectations over $\theta^{i}, i=$ $1,2, . ., n$, yields the expected profits as a function of a particular organizational form $(p, t)$. The next lemma provides a tractable expression for this expected profit function, which is key in the characterization of organizational design.

Lemma 2. The expected profits for a given organizational design $(p, t)$ are given by

$$
\begin{aligned}
\Pi(p, t) & \equiv-E\left[\sum_{i} \min _{\bar{a}^{i}} C^{i}\left(\bar{a}^{i}, p, t \mid \theta_{i}\right)\right] \\
& =n \phi\left(\frac{\phi}{\phi+B}\right) \sigma_{\theta}^{2}-n \phi \sigma_{\theta}^{2}-n(n-t) \delta g(p)-n h(t, \alpha)
\end{aligned}
$$

\section{ORGANIZATIONAL DESIGN}

\section{IV.A The Economics of Coordination}

Increasing returns to ex post coordination. - Organizations can coordinate their activities ex ante by letting employees tightly stick to some pre-agreed course of action. Alternatively, employees may be given substantial discretion to adapt their tasks to local circumstances, in which case broad job definitions (task bundling) and horizontal communication between employees must ensure that tasks are well coordinated. We refer to the latter as ex post coordination.

In contrast to ex ante coordination, a better ex post coordination increases expected profits both through a better coordination of actions and by increasing the adaptation to local circumstances. In the expression for expected profits, (8), the benefits of a better ex post coordination come in the form of a lower $B=\beta(n-t)(1-p)$. An important implication of (8) is that the worse actions are coordinated ex post, the lower are the incentives to locally improve ex post coordination by either increasing task bundling $(t)$ or improving horizontal communication $(p)$. Indeed, the first term of $(8)$, which reflects how well the organization is 
coordinated and adapted, exhibits increasing returns to ex post coordination:

$$
\frac{\partial \Pi(p, t)}{\partial(-B)}=n\left(\frac{\phi}{\phi+B}\right)^{2} \sigma_{\theta}^{2} \quad \text { is decreasing in } B=\beta(n-t)(1-p)
$$

Intuitively, as ex post coordination improves ( $B$ decreases), it is optimal for the organization to become more adaptive and increase employee discretion. Conversely, any increase in employee discretion increases the demand for a better ex post coordination. It follows that any increase in $t$ or $p$, through its impact on employee discretion and the demand for coordination, makes it even more desirable to further increase either task-bundling or the quality of the horizontal communication.

A direct consequence of the above increasing returns to ex post coordination is that unless there are also increasing costs to ex post coordination, organizations will either be extremely rigid and fully rely on ex ante coordination, or they will exhibit substantial employee discretion and rely on extensive ex post coordination (task-bundling and/or intensive communication). Even a very small change in an exogenous parameter, say $\phi$, may then result in dramatic and discrete organizational change. We only expect to see intermediate values of task-bundling or horizontal communication if either $h_{t t}>>0$ for some values of $t$ or $g_{p p}>>0$ for some values of $p$. The next example shows a simple economy where a small change in the importance of adaptation results in a large swing in organizational design.

Example 1. Assume that

$$
h(t, \alpha)=\alpha_{0} t+\frac{\alpha_{1}}{n-t} \quad \text { and } \quad g(p)=\frac{p^{1+\eta}}{1+\eta} \quad \text { with } \quad \alpha_{0}=\alpha_{1}=\frac{1}{4} \quad \text { and } \quad \eta=1.5 .
$$

In addition, let $n=12, \delta=1.85, \beta=1$, and $\sigma_{\theta}^{2}=2$. Notice that $-h(t, \alpha)$ is concave in $t$, that is, it is increasingly costly to bundle tasks, and that complete bundling is prohibitively costly. Still, as mentioned above, there are increasing returns to ex-post coordination, and this induces enough convexity in the profit function so as to yield extreme organizational forms as a function of $\phi$. Indeed a simple numerical computation shows that, in this case,

$$
t^{*}=\left\{\begin{array}{lll}
1 & \text { for } & \phi<3.72 \\
6 & \text { for } & \phi \geq 3.72
\end{array}\right.
$$

Hence, small changes in $\phi$ can result in big changes in organizational design.

Complementarities in organizational design. - In many organizational problems, it is often prohibitively costly to achieve complete task bundling or perfect horizontal communication. As we will argue next, if there is an interior solution for $p^{*}$ and $t^{*}$, high-quality 
communication and substantial task-bundling are complementary instruments of ex post coordination. Hence, we will see more task bundling if the organization can simultaneously improve communication between unbundled tasks. Similarly, the choice variables $p$ and $t$ will move up or down together in response to changes in exogenous parameters, yielding a number of robust comparative static predictions. For example, if the returns to specialization decrease due to information technology or the availability of a higher skilled work-force, then this will result in an increase in both $p$ and $t$.

The intuition for this result rests again on the increasing returns to ex post coordination that characterize organizational design. In particular, any change in one organizational design variable, $p$ or $t$, towards a better ex post coordination, increases the returns to also improve coordination using the other organizational design variable.

Consider first, an increase in task-bundling. The lower the level of specialization, the better tasks are coordinated ex post and, hence, the higher will be the equilibrium level of employee discretion and adaptation. This, in turn, increases the overall demand for coordination, making it optimal to also improve the quality of communication between unbundled tasks.

The impact of a better quality of horizontal communication on the incentives for taskbundling is more intricate. To build intuition, it is instructive to neglect integer constraints on task-bundling and examine the cross-partial of $p$ and $t$, which is given by ${ }^{10}$

$$
\begin{aligned}
\frac{\partial^{2} \Pi(p, t)}{\partial t \partial p} & \equiv n \delta g_{p}-n \beta\left(\frac{\phi}{\phi+B}\right)^{2} \sigma_{\theta}^{2}+2 n \beta \phi^{2} \frac{B}{(\phi+B)^{3}} \sigma_{\theta}^{2} \\
& =\left(\frac{1}{n-t}\right) \frac{\partial \Pi(p, t)}{\partial p}+2 n \beta \phi^{2} \frac{B}{(\phi+B)^{3}} \sigma_{\theta}^{2} .
\end{aligned}
$$

The first two terms in (9) indicate how an increase in the quality of a communication channel affects the incentives to substitute this communication channels through task-bundling. On the one hand, the higher the quality of communication, the lower are the incentives to make communication "perfect" by bundling tasks (second term). Improving communication quality is costly, however. Agents, for example, may be forced to spend half of their time in meetings. Hence, for a given communication technology, the higher the quality of communication, the more the organization saves on communication costs by bundling two tasks (first term). While the former effect may dominate if $p$ is very small, these two effect exactly cancel out in equilibrium. Indeed, as shown in (10), the first order condition with respect to $p$ is proportional to the first two terms.

\footnotetext{
${ }^{10}$ This shortcut is simply to build intuition using standard calculus tools. The Appendix contains a complete treatment where $t \in \mathcal{J}$.
} 
In equilibrium, only the last term in (9) matters then. This term indicates how an increase in the quality of the communication channel between two particular tasks affects the incentives to bundle these tasks with other unbundled tasks. Here, the logic of increasing returns to coordination kicks in again. As two tasks become better coordinated due to improved communication, it is optimal to allow employees more discretion in carrying out these tasks. This, in turn, increases the demand for coordination and the incentives to bundle these tasks with other tasks.

We now formalize the comparative static implications of the complementarity communication quality and task-bundling. To focus on interior solutions, we make the following assumption which guarantees that there is always such a unique (interior) solution for the quality of communication:

A1 The expected profit function $\Pi(p, t)$ is strictly quasi-concave in $p^{11}$

Proposition 3 Given assumption A1:

(i) The equilibrium values of the organizational design variables $p$ and $t$ and the equilibrium level of employee discretion $\sigma^{2}\left(a^{i i}\right)$ are monotone non-decreasing in the parameters $\sigma_{\theta}^{2}$ and $\phi$, and monotone non-increasing in the parameter $\alpha$.

(ii) $p^{*}$ is strictly increasing in $t^{*}$ following any change in $\sigma_{\theta}^{2}, \phi$ or $\alpha$.

(iii) There exists an $\varepsilon>0$ such that whenever $\left|\beta-\beta^{\prime}\right|<\varepsilon$, then $t^{*}(\beta)>t^{*}\left(\beta^{\prime}\right) \Rightarrow$ $p^{*}(\beta)>p^{*}\left(\beta^{\prime}\right)$

Thus, our model predicts that if adaptation to local circumstances becomes more important or the variability of this local environment increases, this will result in more employee discretion, broader job definitions, and better horizontal communication. Similarly, a decrease in the returns to specialization results, trivially, in broader job descriptions, but also more intensive horizontal communication, and increased employee discretion. Moreover, because of the complementarity between these organizational design variables, each change in one of them will reinforce further changes in the others. In contrast, and as we discuss further below, the comparative statics with respect to the importance of task interdependence, as measured by $\beta$, are ambiguous, but task-bundling and communication quality always move up and down together in equilibrium.

\footnotetext{
${ }^{11}$ Obviously, this condition will always be verified whenever the expect profit functions is strictly concave. A (very) sufficient condition for strict concavity is that $2 \beta^{2} \sigma_{\theta}^{2}<\phi \delta g_{p p}(p)$, which will always be verified whenever $\phi$ is large enough, or $\beta$ is small enough.
} 
The next example illustrates the impact of the complementarity between task bundling and the equilibrium level of horizontal communication on organizational design.

Example 2. Assume now that

$$
h(t, \alpha)=\frac{\alpha}{n-t} \quad \text { with } \quad \alpha=1 .
$$

The rest of the parameters, as well as $g(p)$, are as in Example 1. Figure I shows, the optimal task bundling in two cases. The line labeled " $p$ endogenous" shows $t^{*}$ as a function of $\phi$ when the quality of communication, $p$, is optimally chosen. The line labeled " $p$ exogenous" denotes $t^{*}$ when instead the quality of horizontal communication is exogenously set to be equal to $p^{*}(t=1, \phi=2)$, and hence task bundling is the only organizational design variable.

As illustrated in Figure I, because of the complementarity between $t$ and $p$, the returns to increasing task-bundling are much larger if the organization can simultaneously improve communication between unbundled tasks. Similarly, we see intermediate values of specialization for a much smaller range of $\phi \mathrm{s}$ if $p$ is endogenous. The reason is that if $p$ is exogenous, the increasing returns to task-bundling are much smaller as improvements in ex post coordination are much more limited.

One may wonder whether Proposition 3 stems from $\Pi(p, t)$ being supermodular in its arguments, $p$ and $t$, and the exogenous parameters $\sigma_{\theta}^{2}, \phi$ and $-\alpha$. Roughly speaking, a function is supermodular if the returns to increasing one of the arguments are higher the higher the other arguments. Supermodularity then formalizes the idea of 'complementarity' or 'fit' among choice variables and allows for unambiguous comparative statics. ${ }^{12}$

However, $\Pi(p, t)$ is not supermodular on the full support of its variables. As argued above, if the quality of communication is (inefficiently) low, broader task assignments often become more attractive as communication deteriorates: $p$ and $t$ are typically substitutes for $p<<p^{*}$. The complementarity between $p$ and $t$ only holds for equilibrium levels of communication. Therefore, in order to prove Proposition 3, the appendix considers the optimized value of profits with respect to $p$, where $p$ is restricted by a lower bound $\widehat{p}$,

$$
\pi(\widehat{p}, t)=\max _{p \geq \widehat{p}} \Pi(p, t)
$$

\footnotetext{
${ }^{12}$ In particular, if a function of a vector of choice variables $y=\left(y^{1}, \ldots, y^{k}\right)$ and an exogenous parameter $\tau$ is supermodular, then the maximizers $y^{*}(\tau)$ will be monotone nondecreasing in the parameter $\tau$. For an excellent discussion of supermodularity, see Milgrom and Roberts (1990).
} 
and shows that $\pi(\widehat{p}, t)$ is supermodular in $p, t$ and any parameter $\tau \in\left\{\sigma_{\theta}^{2}, \phi,-\alpha\right\}$. Obviously, this change of variables leaves the optimal value of $t$ unchanged. Moreover, if for any fixed values of the other variables, $p^{*}$ is unique, the highest optimal value of $\widehat{p}$ equals $p^{*}$.

\section{IV.B. The Tenuous Trade-off between Coordination and Specialization}

We now consider the impact of two parameters which directly affect the trade-off between specialization and coordination: $\beta$, which characterizes the task interdependence or the importance of coordination and $\delta$, which characterizes the cost of improving the communication technology. We show that whereas the elasticity of the demand for coordination resulted in monotone comparative statics with respect to $\phi$ (importance of adaptation), $\sigma_{\theta}^{2}$ (variance of local circumstances) and $\alpha$ (returns to specialization), this same elasticity of the demand for coordination implies that the impact of $\beta$ and $\delta$ on task specialization is decidedly ambiguous.

Task Interdependence.- Adam Smith's famous observation that the "the division of labor is limited by the extent of the market," has been challenged by both the management literature as well as economists such as Becker and Murphy (1992). These two strands of the literature have emphasized that perhaps, more importantly, specialization is mainly constrained by the need to coordinate activities. In particular, a straightforward comparative static prediction in Becker and Murphy (1992) is that as coordination costs increase, one should see less specialization. Taking into account the endogeneity of the demand for coordination, however, yields a much more subtle picture.

An increase in the importance of coordination affects two margins: First, for a given level of employee discretion, it will become more important to improve ex post coordination by increasing task bundling and improving horizontal communication. This is the effect emphasized in the literature. Second, and as important, it will become optimal to constrain employee discretion, reducing the demand for ex post coordination. Because of the elasticity of the demand for coordination, task specialization may therefore increase as coordination becomes more important.

In particular, if the demand for coordination is sufficiently elastic, the impact of task interdependence on task specialization is non-monotonic. Obviously, if $\beta$ is small and tasks are virtually independent, task coordination can be neglected and it is optimal to have full task specialization $\left(t^{*}=1\right)$ and extreme employee discretion. As $\beta$ increases, it will initially be optimal to reduce specialization $\left(t^{*}>1\right)$ and have broader tasks in order to better coordinate these discretionary actions. As $\beta$ increases further, however, employee discretion becomes more and more limited and eventually it will become optimal to reduce task bundling and 
horizontal communication. Intuitively, if $\beta$ is large, then the decrease in employee discretion resulting from a further increase in $\beta$ outweighs the need to better coordinate whatever is left of employee discretion. The organization then increasingly relies on ex ante coordination as opposed to ex post coordination. In the limit, as coordination becomes very important, the organization reverts again to complete specialization $\left(t^{*}=1\right)$ and fully coordinates activities ex ante by restricting employee discretion. The next example illustrates this logic.

Example 3. Consider Example 2 again, but now rather than exploring the comparative statics with respect to $\phi$ we do so with respect to $\beta$. Figure II illustrates the equilibrium task specialization and communication quality (Panel A), and the equilibrium level of employee discretion (Panel B) as a function of $\beta$, when $\phi=3$. As argued above, once employee discretion is sufficiently limited, any further increase in coordination costs results in more specialization. Note also that $p^{*}$ and $t^{*}$ move up and down together in response to changes in $\beta$, as predicted by Proposition 3, (iii).

More formally, neglecting integer constraints, the cross partial with respect to $\beta$ and $t$ is given by:

$$
\frac{\partial^{2} \Pi(p, t)}{\partial t \partial \beta}=n(1-p)\left(\frac{\phi}{\phi+B}\right)^{2} \sigma_{\theta}^{2}-2 n(1-p) B \frac{\phi^{2}}{(\phi+B)^{3}} \sigma_{\theta}^{2}
$$

which is positive if and only if $\phi>B$. In other words, fixing $\phi$, if task coordination becomes more important, this increases local incentives towards more specialization if and only if employee discretion, given by $(\phi /(\phi+B))^{2} \sigma_{\theta}^{2}$, is sufficiently limited.

Since the non-monotonicity stems from the elasticity of the demand for coordination, it will only occur if $\phi$ is not too large. Indeed, if adaptation to local circumstances is very important, employee discretion will vary little with task interdependence and the demand for coordination is rather inelastic. We formalize the above intuitions as follows. Define $\hat{t}$ as the optimal level of task-bundling if the organizations wants to achieve perfect communication between different tasks

$$
\hat{t} \equiv \arg \min _{t}\{n(n-t) \delta g(1)+h(t, \alpha)\} .
$$

Then the following result holds.

Proposition 4. (a) Given $\alpha$, there exists a unique $\bar{\phi}$ such that

$$
\begin{array}{llll}
\lim _{\beta \longrightarrow \infty} t^{*}=\hat{t} & \text { if } & \phi>\bar{\phi} \\
\lim _{\beta \longrightarrow \infty} t^{*}=1 & \text { if } & \phi<\bar{\phi}
\end{array}
$$

where $t^{*}$ is the optimal number of tasks per job, and $(b) \bar{\phi}$ is increasing in $\alpha$. 
The following corollary is then immediate and given without proof.

Corollary 5. If $\phi<\bar{\phi}$, then the level of task specialization is non-monotonic in the degree of task-interdependence $(\beta)$.

Communication costs. - Perhaps the most natural way to coordinate specialized activities is through communication. Indeed, agents spend a substantial amount of their time communicating with each other in the workplace and meetings, conference calls, and writing and reading electronic mail dominate the modern professional environment. An important question therefore is how improvements in communication technology or differences in the ease of communication affect the specialization of agents. For instance, if due to logistical or epistemological reasons, the transmission of information is easier in certain economic processes or activities, do we expect to see more or less specialization?

Bolton and Dewatripont (1992) and Garicano (2000), among others, have addressed this issue in different settings. ${ }^{13}$ These authors identify an important trade-off between specialization and communication cost, namely, that one should expect to see more specialization as communication costs decrease and coordination becomes easier. But, as before, taking into account the endogeneity of the demand for coordination yields a much more subtle picture.

In very much the same way as a change in $\beta$, a decrease in the cost of (improving) communication, as characterized by the parameter $\delta$, affects two margins. First, for a given level of employee discretion and adaptation, it will be easier to coordinate specialized activities, reducing the need for task-bundling. This is the effect emphasized by Bolton and Dewatripont (1992) and Garicano (2000). Second, as communication improves it will become optimal to become more adaptive and increase employee discretion, which favors more task bundling in order to reduce coordination failures. Because of the elasticity of the demand for coordination, task specialization may therefore decrease as communication costs decrease or communication technology improves.

Consider, for example, a case in which communication is very poor or costly $(\delta$ is very large), and complete task bundling is not feasible. Organization then often forego the benefits of being adaptive, but instead choose to limit the discretion of employees and reap the benefits of extreme task specialization. In the latter case, lower communication costs will typically be complementary to broader task assignments. In particular, improvements in communication

\footnotetext{
${ }^{13}$ Bolton and Dewatripont (1992) consider the returns to division of labor in information processing. Garicano (2000) considers vertical specialization in knowledge acquisition, where some agents may specialize in solving more difficult problems.
} 
technology may transform this very rigid and specialized organization into a flexible organizations characterized by substantial employee discretion and broad task assignments and intensive horizontal communication. As communication technology further improves, however, the benefits of task bundling in terms of reduced coordination failures or lower communication costs become smaller and smaller. Task bundling and improvements in communication technology become then substitutes and task specialization increases again. The following example illustrates the non-monotonicity of organizational design with respect to $\delta$.

Example 4. Consider again Example 1, but now the comparative statics are with respect to $\delta$ (cost of communication) rather than $\phi$, so we set $\phi=3$. Figure III plots $p^{*}$ as a function of $\delta$. The figure also denotes the degree of task bundling, $t^{*}$, as a function of $\delta$. Reading the figure from right to left, as $\delta$ falls below 1.365, the organization is transformed from a very rigid and specialized organization to an organization with substantial task-bundling, intensive horizontal communication and almost unlimited employee discretion. This illustrates how for large communication costs, improvements in communication technology and task-bundling are complements. Note that even when communication becomes perfect $\left(p^{*}=1\right)$, there is still a role for task-bundling in reducing communication costs. Only when the communication cost parameter $\delta$ falls below 0.59 , communication has become sufficiently cheap to warrant full specialization. In this example, task-bundling and improvements in communication technology are thus only substitutes when communication costs are small.

The following proposition gives sufficient conditions under which the impact of a better communication technology has a non-monotonic impact on task specialization. Since the nonmonotonicity stems from the elastic demand for coordination, it will only be observed if $\phi$ is not too large.

Proposition 6. Given $\alpha$ there exists a unique $\bar{\phi}$ such that

$$
\lim _{\delta \longrightarrow \infty} t^{*}=\lim _{\delta \longrightarrow 0} t^{*}=1 \quad \text { if and only if } \quad \phi<\bar{\phi}
$$

where $\bar{\phi}$ is increasing in $\alpha$.

Then, as before, the following corollary follows immediately and is given without proof.

Corollary 7. For $\phi<\bar{\phi}$, the level of task specialization is non-monotonic in the cost of communication $\delta$. 


\section{EXTENSIONS}

\section{V.A The Role of Management}

"The key role of management in organizations is to ensure coordination."

Milgrom and Roberts (1992), Chapter 4.

A key insight in the previous analysis was that organizations can rely either on ex ante coordination, where specialized employees stick tightly to some pre-agreed task rules, or ex post coordination, where broadly defined job definitions and intensive horizontal communication coordinate discretionary actions. Obviously, the role of management in organizations is complex and varied but coordination, as emphasized by Milgrom and Roberts (1992), is a central aspect of this role. In particular, in this section, we posit that the role of management lies in improving ex ante coordination: management updates and improves the quality of the task rules to which employees stick in the absence of horizontal communication.

A direct consequence of this role is that a large management and extensive vertical coordination will be complementary to specialized jobs and limited horizontal communication. In contrast, a small management will be complementary to broadly defined jobs and intensive horizontal communication. To put it differently, an organization can adapt while remaining coordinated by increasing employee discretion and having broadly defined jobs. Alternatively, the organization can remain adaptive and coordinated by having its managerial force intervene frequently in order to keep up-to-date the task guidelines which very specialized agents use to coordinate their activities. Both 'types' of organizational design aim at achieving adaptation through a set of very different but complementary and mutually reinforcing elements of organizational design.

\section{V.A.1 What do managers do?}

In the absence of informative communication, agents behave as if the co-worker in charge of task $i$ has chosen a primary action $a^{i i}$ equal to average state of nature $\widehat{\theta}^{i}$. Knowledge of $\widehat{\theta}^{i}$, the rule that agents follow in the case of faulty communication, is thus key to guarantee efficient coordination in the workplace. But, where do these rules come from? In this section, we propose a simple dynamic version of our model in which the role of management is exactly that: introducing and improving these rules. ${ }^{14}$

\footnotetext{
${ }^{14}$ Organizational instructions include the training which employees receive, routines and procedures developed by management, task manuals, etc.
} 
Formally, we consider a two period model, in which in period $2, \theta^{i}$ is drawn from a distribution with mean $\hat{\theta}^{i}+\varepsilon^{i}$, where $\varepsilon^{i}$ is an i.i.d shock, normally distributed with mean $E\left(\varepsilon^{i}\right)=0$ and variance $\sigma_{\varepsilon}^{2}$, which occurs after period $1 .{ }^{15}$ Thus, the unconditional variance of $\theta^{i}$ in the second period equals $\sigma_{\theta}^{2}+\sigma_{\varepsilon}^{2}$. As before, only the employee in charge of task $i$ can observe $\theta^{i}$. Management, in contrast cannot but it does observe $\varepsilon^{i}$ and hence it has a better estimate of the mean realization of the local circumstances in period $1, E\left[\theta^{i} \mid \varepsilon^{i}\right]$, than workers, $E\left[\theta^{i}\right]$. Management then may choose to intervene in period 1 to improve the rules workers will follow in the absence of information.

For simplicity, we assume that when instructions for a particular primary action are improved upon, they are also improved for the actions complementary to this primary action. If no new instructions are issued, the old instructions remain in place. Naturally, communicating and explaining new guidelines to employees is costly. Let

$$
I(m) \quad \text { with } \quad I_{m}<0
$$

be the cost of changing the rules for a vector of actions $\bar{a}^{i}$, where $m$ is the size of management in the organization, an endogenous variable. ${ }^{16}$ Thus, $I(m)$ measures the ability of the organization to react to aggregate events in a centralized way and the larger the number of managers in the organization the lower the costs of implementing change. ${ }^{17}$

\section{V.A.2 Management intervention}

Organizational design will take place taking into account the possibility of management intervention to improve coordination and organizational adaptability. Lemma 7 shows that, quite naturally, management will implement change whenever the shock $\varepsilon^{i}$ is sufficiently large.

Lemma 8. (a) Management updates organizational guidelines in period 2 from $\widehat{\theta}^{i}$ to $\widehat{\theta}^{i}+\varepsilon^{i}$ whenever $\left|\varepsilon^{i}\right| \geq b^{*}$ where

$$
b^{*}=\left[\left(\frac{\phi+B}{\phi B}\right) I(m)\right]^{1 / 2} .
$$

(b) $b^{*}$ is increasing $p$ and decreasing in $m$ and $\phi$. Moreover, $b(\bar{t})>b(\underline{t})$ for $\underline{t}, \bar{t} \in \mathcal{J}$ and $\underline{t}<\bar{t}$.

\footnotetext{
${ }^{15}$ The assumption on the normality of $\varepsilon^{i}$ is made to simplify the proofs of the propositions and it is much stronger than needed.

${ }^{16}$ In contrast to horizontal communication, the outcome of vertical communication is thus deterministic, which simplifies the analysis. It is easy to see, however, that as long as the probability of success is correlated, our results would not be affected if the outcome was probabilistic.

${ }^{17}$ For simplicity, and without any loss of generality we take $m \in \mathbb{R}_{+}$.
} 
First, trivially, intervention by management is less likely the lower the size of its force, $m$, as the cost of intervention $I(m)$ is decreasing in it. Second, intervention by management is less likely the larger the quality of the horizontal communication and the more tasks are bundled. If $p$ is high, then management can rely on the communication across tasks to implement the necessary coordination, which makes the introduction of a new organizational rule $\widehat{\theta}^{i}+\varepsilon^{i}$ less useful. Similarly, the higher the number of tasks per job, the more adaptive the organization will be and hence the less useful adaptation is via management intervention.

As in Section III knowledge of the expected profit function is needed in order to characterize organizational design. Let $P$ be the probability that management intervenes in a particular task, that is

$$
P=\operatorname{prob}\left[\left|\varepsilon^{i}\right|>b\right] .
$$

The following lemma provides the expected profit function in the second period, that of the first period being identical to the one reported in Lemma 2. For analytical purposes, we will consider $b$ as an organizational design variable, chosen at the organizational design stage. In equilibrium, however, $b$ will always satisfy equation (11).

Lemma 9. The expected cost function for task $i$ in period 2 is given by

$$
\begin{aligned}
& \Pi(p, t, b,-m) \equiv-E\left[\sum_{i=1}^{n} \min _{\bar{a}^{i}} C^{i}\left(\bar{a}^{i}, p, t, b,-m \mid \theta^{i}, \varepsilon^{i}\right)\right] \\
& \quad=-\delta n(n-t) g(p)-n\left(\frac{\phi B}{\phi+B}\right)\left[\sigma_{\theta}^{2}+\sigma_{\varepsilon}^{2}\right] \\
& -n \omega m-n P\left[I(m)-\left(\frac{\phi B}{\phi+B}\right) E\left[\varepsilon^{i^{2}}|| \varepsilon^{i} \mid>b\right]\right] .
\end{aligned}
$$

The term (12) represents the expected second period cost in the absence of any coordination by management. It is identical to the organizational cost expression in Lemma 2, except that the variance of $\theta^{i}$ now equals $\sigma_{\theta}^{2}+\sigma_{\varepsilon}^{2}$. Expression (13) represents the impact of management on the organization's profit function. First, the presence of management involves a fixed cost $\omega m$, where $\omega$ stands for the manager's wage per task. Second, whenever management intervenes to update $\widehat{\theta}^{i}$, which occurs with probability $P$, the organization suffers a variable cost $h(m)$ but it reduces the "variance" by a term that is proportional to $E\left[\varepsilon^{i^{2}}|| \varepsilon^{i} \mid>b\right]$; it is as if the second period variance of $\theta^{i}$ is only $\sigma_{\theta}^{2}$ as opposed to $\sigma_{\theta}^{2}+\sigma_{\varepsilon}^{2}$. 


\section{V.A.3 Management and Organizational Design}

We are now ready to characterize the relationship between the size of management, the frequency of managerial intervention, and the other organizational design variables. As before, we consider the expected profits of a particular organizational form as a function of parameters $\tau \in\left\{\phi, \alpha, \beta, \delta, \omega, \sigma_{\theta}^{2}, \sigma_{\varepsilon}^{2}\right\}$ and with some abuse of notation we denote it $\Pi(p, t, b,-m, \tau)$. As in Section III we focus on the case of interior solutions, and make a similar assumption in terms of the strict quasiconcavity of the profit function.

A2 The profit function $\Pi(p, t, b,-m, \tau)$ is strictly quasi-concave in $p$.

Finally, for the same reasons as above, the complementarity between $p$ and $t$ only holds for equilibrium levels of communication. Hence we restrict our analysis to the function,

$$
\pi(\widehat{p}, t, b,-m, \tau)=\max _{p \geq \widehat{p}} \Pi(p, t, b,-m, \tau),
$$

rather than to $\Pi(p, t, b,-m, \tau)$. Then we can prove the following

Proposition 10. (i) The expected profit functions

$$
\pi(\widehat{p}, t, b,-m,-\alpha), \pi(\widehat{p}, t, b,-m, \omega), \text { and } \pi\left(\widehat{p}, t, b,-m, \sigma_{\theta}^{2}\right)
$$

are supermodular on $\mathbb{R}_{+} \times \mathcal{J} \times \mathbb{R}_{+}^{3}$.

(ii) The equilibrium values of the organizational design variables $p, t, b$ and $-m$ are monotone non-decreasing in the parameters $\sigma_{\theta}^{2}$ and $\omega$, and monotone non-increasing in $\alpha$.

In a nutshell, the size of management, the extent of ex-ante coordination in the organization, is a substitute for the equilibrium level of employee discretion, or the extent of ex-post coordination. To put it differently, the returns to increasing the size of management $(m)$ and the frequency of managerial intervention (as measured by $b$ ) are increasing in the level of task specialization and decreasing in the quality of the horizontal communication network. Proposition 9 coincides with the casual observation that organizations with extensive management forces are associated with the intense specialization of the workers' narrow job descriptions. ${ }^{18}$

\footnotetext{
${ }^{18}$ Some empirical evidence on the dissapperance of middle management has been recently reported in Rajan and Wulf (2002). Consistent with our complementarity result, this 'flattening of the hierarchy' occured simultaneously with changes in workplace organization towards broader job definitions and team work.
} 
The most interesting comparative static results of Proposition 9 are those with respect to task uncertainty. In particular, an increase in the local uncertainty $\sigma_{\theta}^{2}$ is met by organizations with a reduction in the size of the managerial force, $m$, and a decrease in the frequency of vertical coordination, that is, an increase in the intervention bound $b$. The reason is that an increase in local uncertainty makes it optimal to increase task bundling, which decreases the returns to vertical coordination. In contrast, an increase in the overall uncertainty which is not related to local uncertainty, $\sigma_{\varepsilon}^{2}$, has ambiguous implications. Indeed, the direct effect of an increase in $\sigma_{\varepsilon}^{2}$ will be both to increase management and task bundling to improve adaptation. More task-bundling and a larger management, however, are substitutes: increasing one lowers the returns of increasing the other. Therefore, an increase in $\sigma_{\varepsilon}^{2}$ may result in a decrease in the size of the managerial force, $m .{ }^{19}$

\section{V.B Endogenous Ability to Adapt and Investments in Skills and IT}

In our basic model, adaptation is uniquely constrained by the organization's ability to coordinate. In many circumstances, though, adaptation to local conditions may require specific knowledge, skills or supporting tools. In particular, a number of studies (Autor, Levy, and Murname (2003) and Maurin and Thesmar (forthcoming)) have argued that the demand for high-skilled labor is a demand for employees which possess superior cognitive or informationprocessing abilities. In terms of our model, the cognitive abilities of higher skilled workers may allow the latter to better observe local conditions or they may better know how to adapt to these local conditions. Similarly, organizations may improve the ability of its employees to adapt by investing in Information Technology (IT), such as expert systems and database technology or by providing more training.

To formalize the notion of an endogenous ability to adapt, we assume that an agent in charge of task $i$ observes $\theta^{i}$ with probability $q(t, x)$, whereas with probability $1-q(t, x)$, he observes pure noise. The variable $x$ denotes these investments in skills and IT, where we assume that $q_{x}(t, x)>0$ and $q_{x x}(t, x)<0$. We denote by $C(x, \lambda)$ the related costs to the organization with $C_{x}(0)=0, C_{x}>0$ and $C_{x x}>0$. The parameter $\lambda$ could, for example, reflect the cost of IT equipment in which case it is naturally to assume that $C_{x \lambda}(x, \lambda)>0$.

So far, we have modeled the returns of specialization uniquely in terms of reduced labor costs to complete a particular task. Task specialization, however, may not only reduce the labor cost of performing a task, it may also improve the quality of task execution itself. In particular, for a given skill and IT stock, an increase in task-specialization may potentially

\footnotetext{
${ }^{19}$ This could occur, for example, if $I(m)$ becomes very convex for larger values of $m$.
} 
improve the ability of an employee to observe or adapt to local circumstances. We assume therefore that

$$
q(\bar{t}, x) \leq q(\underline{t}, x) \quad \text { for } \quad \bar{t}, \underline{t} \in \mathcal{J} \quad \text { with } \quad \bar{t}>\underline{t},
$$

or, ignoring integer constraints, $q_{t} \leq 0$. Finally, it is natural to assume that investments in skills and IT are at least weakly more productive in those case where extensive task-bundling has decreased the employees ability to adapt. Neglecting integer constraints, this is equivalent to assuming that $q_{x t}(t, x) \geq 0$. The management literature, for example, has argued that improvements and investments in IT have made the informational returns to specialization, as expressed by $-q_{t}$, much less relevant than they used to be. ${ }^{20}$

For a given $q$, the choice of primary action for the employee in charge of task $i$ is then

$$
a^{i i}=\left\{\begin{array}{cll}
\hat{\theta}^{i}+\left(\frac{\phi}{\phi+B}\right)\left(\theta^{i}-\widehat{\theta}^{i}\right) & \text { when } \theta^{i} \text { observed } & \text { (probability } q(t, x)) \\
\widehat{\theta}^{i} & \text { when } \theta^{i} \text { not observed } & \text { (probability } 1-q(t, x))
\end{array},\right.
$$

and the complementary actions are as in Lemma 1. The expected profit associated with organizational design $(p, t)$ is derived now along very similar lines as before and can be written as

$$
\Pi(p, t)=n q(t, x)\left(\frac{\phi^{2}}{\phi+B}\right) \sigma_{\theta}^{2}-n \phi \sigma_{\theta}^{2}-\delta n(n-t) g(p)-n h(t, \alpha)-C(x, \lambda) .
$$

Maximizing the expected profits with respect to $x$ then yields

$$
q_{x}\left(t, x^{*}\right)\left(\frac{\phi^{2}}{\phi+\beta(1-p)(1-t)}\right) \sigma_{\theta}^{2}=C_{x}\left(x^{*}, \lambda\right)
$$

It follows that equilibrium investments in skills and IT are strictly increasing in both $p$ and $t$, even when $q_{x t}(t, x)=0$. Intuitively, the larger are $p$ and $t$, the better the organization can coordinate discretionary behavior, and hence, the more worthwhile it is to invest in skills and IT which allow employees to better observe or adapt to local circumstances. Conversely, the higher the skill-level of employees or the stock of flexibility enhancing IT, the larger are the payoffs to improve ex-post coordination. In other words, $x$ is complementary with $t$ and $p$. If one neglects any direct impact of task specialization on the ability to adapt, that is $q(t, x) \equiv q(x)$, this yields the following comparative static implications:

\footnotetext{
${ }^{20}$ Hammer and Champy (2001), for example, recount how the development of a sophisticated computer system designed to support deal structures, allowed IBM Credit to replace several specialists - credit checkers, pricers, and so on - with one generalist. All tasks fell well within the capability of a single individual when supported by a computer system providing access to the data and tools the specialists would use.
} 
A3 The expected profit function $\Pi(p, t, x)$ is strictly quasi-concave in $p$.

Proposition 11 Assume $q(t, x) \equiv q(x)$ and $A 3$, then

(i) The equilibrium values of the organizational design variables $x, p$ and $t$ are monotone increasing in $\sigma_{\theta}^{2}$ and $\phi$, and monotone decreasing in $\alpha$ and the parameter $\lambda$, which characterizes the cost of information technology equipment

(ii) The equilibrium ability to observe or adapt to local circumstances, $q\left(x^{*}\right)$, is strictly increasing in $t^{*}$ following any change in $\sigma_{\theta}^{2}, \phi, \alpha$ or $\lambda$.

From Proposition 11, if the returns to specialization come uniquely in terms of reduced labor costs, then the complementarity between task-bundling and investments in skills and IT implies that an increase in task-bundling indirectly increases the ability of employees to adapt. As argued above, however, task specialization may also improve the quality of task execution by improving the ability of an employee to observe or adapt to local circumstances. The total impact of an increase in $t$ on the ability to adapt is then given by

$$
\underbrace{q\left(\bar{t}, x^{*}(\bar{t})\right)-q\left(\underline{t}, x^{*}(\underline{t})\right)}_{+ \text {or }-}=\underbrace{q\left(\bar{t}, x^{*}(\bar{t})\right)-q\left(\underline{t}, x^{*}(\bar{t})\right)}_{-}+\underbrace{q\left(\underline{t}, x^{*}(\bar{t})\right)-q\left(\underline{t}, x^{*}(\underline{t})\right)}_{+}
$$

and is ambiguous. Put differently, an increase in task-bundling may translate itself mainly in more investments in skills and IT, in which case the ability to adapt is left intact or improved. In contrast, if investment in IT and skills are not very productive, task-bundling will likely be associated with a lower equilibrium ability to adapt.

In the remainder of this section, we neglect any investments in skills or IT, in which case more task-bundling unambiguously reduces the ability of employees to adapt. Nevertheless, we show that as long as the impact of task-bundling on $q(t, x)$ is not too negative, our qualitative results remain valid. In particular, what is needed is that the equilibrium level of employee discretion is increasing in the amount of task-bundling. Assume therefore that $x$ is exogenously fixed, that is $q(t, x) \equiv q(t)$. Then the equilibrium level of employee discretion, as characterized by the variance in the primary actions, is given by

$$
\sigma^{2}\left(a^{i i}\right) \equiv q(t)\left(\frac{\phi}{\phi+B}\right)^{2} \sigma_{\theta}^{2}
$$

and from (14), the equilibrium quality of communication $p^{*}$ satisfies

$$
\frac{\delta}{\beta} g_{p}(p)=\sigma^{2}\left(a^{i i}\right)
$$


It follows that if the assumption $A 1$ above is met, that is, if the profit function (14) is strictly quasiconcave in $p$, then the equilibrium value of $p$ will be increasing in $t$ whenever an increase in $t$ makes the primary actions more adaptive to the local information, that is whenever

$$
\frac{\partial \sigma^{2}\left(a^{i i}\right)}{\partial t}>0 \Leftrightarrow-\frac{q_{t}}{q(t)}<2 \frac{\beta(1-p)}{\phi+\beta(1-p)(n-t)} .
$$

From (16), more task-bundling results in more employee discretion and better communication whenever (i) the informational returns to specialization, as characterized by $-q_{t} / q(t)$, are not too large and (ii) the equilibrium level of communication quality, $p$, is not too high. Intuitively, if $p$ is very large, the factor limiting adaptability is the quality of the information observed by workers, rather than the inability to coordinate. By increasing the degree of specialization the organization will be able to generate better information on the local conditions, with little effect on the organization's ability to coordinate as $p$ is very large. It follows then that for a high levels of $p$, communication and task bundling are substitutes rather than complements.

Condition (16) guarantees robust comparative statics with respect to the 'traditional' returns to specialization $\alpha$. Condition (16) is also sufficient to guarantee increasing returns to ex-post coordination and the ensuing convexity of the profit-function in $t$. In order to obtain robust comparative statics with respect to task-uncertainty, however, a slightly more stringent condition is necessary. As in our basic model, an increase in the variance of local information pushes the organizations to become more adaptive to this local information. Now, however, the organization could potentially become more adaptive by increasing specialization and, hence, improving the observation of local conditions. Concretely, keeping $p$ fixed and neglecting integer constraints, an increase in $\sigma_{\theta}^{2}$ will result in more task-bundling whenever

$$
-\frac{q_{t}}{q(t)}<\frac{\beta(1-p)}{\phi+\beta(1-p)(n-t)}
$$

the interpretation of which is similar to that of condition (16). The following example illustrates the above findings:

Example 5. Assume that

$$
q(t)=e^{-\rho(t-1)} \quad \text { with } \quad \rho>0 .
$$

such that the parameter $\rho$ equals $-q_{t} / q(t)$ and characterizes the returns to specialization. Only if agents are fully specialized they observe the signal perfectly. Under $A 1$, a sufficient condition for (16) and (17) to hold for the equilibrium values of $p^{*}$ and $t^{*}$ is then that 
they hold for $(p, t)=\left(p_{1}, 1\right)$, where $p_{1} \equiv \arg \max \Pi(p, 1)$ is given by: ${ }^{21}$

$$
\frac{\delta}{\beta} g_{p}\left(p_{1}\right)=q(1)\left(\frac{\phi}{\phi+\beta\left(1-p_{1}\right)(n-1)}\right)^{2} \sigma_{\theta}^{2},
$$

For the set of economies for which (16) and (17) hold given $(p, t)=\left(p_{1}, 1\right)$, unambiguous comparative statics with respect to $\alpha, \rho$ and $\sigma_{\theta}^{2}$ obtain, the weaker condition (17) being sufficient for $\alpha$ and $\rho$. In particular, it is straightforward to show that the equilibrium values of $p$ and $t$ and the employee discretion $\sigma^{2}\left(a^{i i}\right)$ are then weakly increasing in $\sigma_{\theta}^{2}$ and weakly decreasing in the traditional and the informational returns to specialization, $\alpha$ and $\rho$, respectively. In addition, $p^{*}$ is strictly increasing in $t^{*}$ following any change in $\sigma_{\theta}^{2}$, $\alpha$ or $\rho$. As noted, it is natural to assume that improvements in information technology are associated with a decrease in $\rho=-q_{t} / q(t)$.

To summarize, if the ability to adapt to local circumstances is both increasing in the specialization of employees and in investments in skills and IT, the total impact of an increase in task-bundling on the ability to adapt to local circumstances is ambiguous. More taskbundling will then most likely result in more employee discretion, in which case our qualitative results are not affected.

\section{V.C The Nature of Communication}

In our model, we have assumed that communication occurs task-to-task: Even if task $j$ and task $k$ are allocated to the same agent, if the latter understands the implications of $a^{i i}$ for task $j$, this does not imply that he understands what it means for task $k$. Given a level of task bundling $t$, each task then requires $(n-t)$ communications, from which the total cost of the organization's communication network equals $n(n-t) \delta g(p)$.

As mentioned above, task-to-task communication is a natural assumption when the way a particular task is carried out cannot be easily summarized and affects different tasks differently. In addition task-to-task communication has the theoretically appealing feature that communication costs between two unbundled task $i$ and $j$ are independent of the degree of specialization. Thus, modeling communication task-to-task does not introduce any organizational design bias.

An alternative assumption about the nature of communication occurs when tasks are sufficiently simple such that they can be comprehensively described in a nut-shell. An employee

\footnotetext{
${ }^{21}$ Indeed, the RHS of the inequality (16) is increasing in $t$ if $p$ is fixed. Moreover, the optimized value of $p$ given $t, p^{*}(t)$, is increasing in $t$ only if the inequality holds strictly. Since from $A 1, p^{*}(t)$ is continuous in $t$, it follows that if the inequality holds for $(p, t)=\left(p_{1}, 1\right)$, then it will hold for any $(p(t), t)$ with $t>1$.
} 
which understands the implication of the choice of the primary action of task $i$ for task $j$ is then also likely to understand the implications for any other task $k \neq j$ allocated to him. We refer to this as task-to-person communication. As already mentioned in Section II though, task-toperson communication introduces a mechanical complementarity between $t$ and $p$. The reason is that a higher level of task-bundling then increases the returns to improve the communication quality $p$ for purely technological reasons: the associated communication cost can be split among more tasks.

Indeed if communication occurs task-to-person then, given a level of task-bundling $t$, each task only requires $\left(\frac{n}{t}-1\right)$ communications, resulting in a total communication cost equal to

$$
n\left(\frac{n-t}{t}\right) \delta g(p)
$$

and expected profits are given by:

$$
\Pi(p, t) \equiv-n \phi\left(\frac{B}{\phi+B}\right) \sigma_{\theta}^{2}-n\left(\frac{n-t}{t}\right) \delta g(p)-n h(t, \alpha)
$$

As one can notice, task-to-person communication introduces a mechanical complementarity between task-bundling and communication quality. A higher level of task-bundling increases the returns to improve the communication quality $p$ for purely technological reasons: the associated communication cost can be split among more tasks. Indeed, neglecting integer constraints on task-bundling, of $p$ and $t$, is now given by

$$
\frac{\partial^{2} \Pi(p, t)}{\partial t \partial p}=\left(\frac{1}{n-t}\right) \frac{\partial \Pi(p, t)}{\partial p}+2 n \beta \phi^{2} \frac{B}{(\phi+B)^{3}} \sigma_{\theta}^{2}+\frac{n(n-t)}{t^{2}} \delta g(p)
$$

The first term equals zero when $p=p^{*}$, the second term represents the complementarity between $p$ and $t$ which stems from the increasing returns to coordination, and the last term represents the mechanical complementarity between $p$ and $t$. Obviously, this mechanical complementarity will only reinforce our results on complementarity between $p$ and $t$, and will not interfere with the non-monotonic comparative static results with respect to $\beta$ and $\delta$. One can therefore easily show that Propositions/Corollaries $3-7$ hold under task-to-person communication.

\section{EVIDENCE AND EXAMPLES}

In this section we survey some of the empirical findings on organizational design and its determinants. We start by reporting evidence concerning recent changes in workplace reorganization. Essentially, this evidence suggests that there has been a trend from one organizational 
mode to another. The first is characterized by stark specialization and centralized decision making whereas the second hinges on broad task assignments and increased worker responsibility. As we have argued, these two modes are the natural result of the endogenous demand for coordination. We then examine the empirical link between organizational design and the relevant parameters in our model, such as the extent of task interdependence, environmental uncertainty, or the information technology.

\section{VI.A The emergence of new workplace practices}

A substantial body of work documents how the organization of work inside firms experienced dramatic changes in recent decades. A number of these accounts are mainly historical. Cyert and Mowery (1987) and Boyer (1991), for example, provide an analysis of the evolution of modern manufacturing in the US and other OECD countries, respectively. They depict how, since the beginning of 20th century, Fordist and Tayloristic principles increasingly defined the organization of the work process. These principles resulted in a deep (and deepening) division of labor as well as in a strong centralization of decision making, where control over the pace of work and the structure of jobs belonged to management. In the 1980s, however, a new model of management and work organization started to emerge, characterized by more decentralization and a weaker division of labor. In this new organizational regime, workers tend to perform a wider range of tasks and are allocated more responsibility.

Caroli and Van Reenen (2001) offer empirical evidence on the trend towards broader tasks assignment and increased worker responsibility, based on the British Workplace Industrial Relations Survey (WIRS). In this survey, senior managers were asked whether organizational change led to more or less responsibility as well as to whether it resulted in a wider range of tasks performed. The results are contained in Table 1, which partially reproduces Table I in Caroli and Van Reenen (2001). 46.2\% and 32.8\% of managers responded that organizational change led to more responsibility in non manual and manual jobs respectively and only rarely did it lead to a reduction of responsibility. Similarly, organizational change resulted in broader task assignments in both non manual (62.5\%) and manual jobs (39.5\%).

In addition, other empirical studies have found that organizational change has led to more worker autonomy and responsibility, ${ }^{22}$ to a stronger emphasis on team work, ${ }^{23}$ and to a greater intensity and variety of communication channels. ${ }^{24}$ Caroli (2001) and the report by OECD (1999) provide extensive surveys of this literature. Consistent with the predictions of

\footnotetext{
${ }^{22}$ See Freechou and Greenan (1995) and Coutrot (1996).

${ }^{23}$ See Osterman (1994), who uses a US survey on innovative work practices, and Coutrot (1996).

${ }^{24}$ See Bue (1989) and Coutrot (1996).
} 
our model, both studies find substantial evidence that these new organizational practices tend to be adopted in clusters, suggesting that they are complementary. ${ }^{25}$

\section{VI.B The impact of Information Technology (IT)}

The potential link between information technology (IT) and new workplace practices has received much attention lately. Brynjolfsson and Hitt (1998), for example, provide substantial evidence that IT and new work practices cluster together. ${ }^{26}$ In particular their focus is on the impact of organizational design on the demand for IT and they find that this is larger for firms with substantial use of teams and broad job definitions. To briefly illustrate some of their results, Table 2, which is taken from their Table 2a, shows the correlation of various measures of information technology with the use of teams and broader job descriptions. Notice that the correlation is positive and significant independently of the measure of IT used. In addition, they also provide evidence that firms with larger investments in IT are more likely to give their workers substantial discretion. ${ }^{27}$ The impact of IT is consistent with our model's predictions. First, as argued in Section VI.B, IT increases the capabilities of employees to perform information processing tasks, which is complementary with broader job assignments and more employee discretion. Second, IT is also likely to reduce communication costs, an effect that the previous literature has argued leads towards more specialization. ${ }^{28}$ This is in direct contradiction with the results in Table 2 that show, for example, that there is a strongly significant positive correlation between the use of email and broadly defined jobs. Our model resolves this apparent contradiction by showing that by increasing employee discretion, lower communication costs often results in more task-bundling, as shown in the data.

\section{VI.C The impact of environmental uncertainty}

New workplace practices can also result from changes in demand conditions. For instance, using US data, Osterman (1994) finds that establishment facing an international demand tend to use more new workplace practices, as are establishment which have adopted a 'high road strategy' emphasizing product quality and customization. To the extent that these conditions are associated with a more volatile demand and consumer needs, this is consistent with the our comparative static predictions with respect to environmental uncertainty and the importance of adaptation.

\footnotetext{
${ }^{25}$ Osterman (1994), Ichinowski et al. (1997), Brynjolfsson et al. (1998) and Bresnahan et al. (2002).

${ }^{26}$ See also, Bresnahan, Brynjolfsson, and Hitt (2002), which provide additional evidence for this complementarity, as well as the link with demand for high-skilled labour.

${ }^{27}$ Similar results are obtained by Coutrot (1996) on French data.

${ }^{28}$ See Bolton and Dewatripont (1992) and Garicano (2000).
} 
A much earlier account of the link between task uncertainty and organizational design can be found in an influential paper by Van de Ven, Delbeq, and Koenig (1976). This paper studies the coordination and communication modes in sixteen district offices as well as the headquarter of a large employment security agency. ${ }^{29}$ They collected observations on 197 formal work units, as officially defined in the organizational chart. These units, which are formed by a supervisor and a varying number of agents, face different coordination and adaptation challenges. Van de Ven et al. (1976) measured the degree of uncertainty that each unit faced ${ }^{30}$ as well as the degree with which different "modes" are used to achieve coordination. According to their classification, coordination can be achieved through the use of "impersonal coordination modes," which include the use of rules and procedures and that of plans and schedules, "personal coordination modes," which include both vertical and horizontal communication, and "group coordination mode," that includes both the use of scheduled and unscheduled meetings.

Table 4, which is taken from Van de Ven et al. (1976), shows the zero order correlation among independent variables and coordination modes. The correlation between task uncertainty and impersonal modes of communication, which includes rules and procedures and plans and schedules, is strongly negative, whereas it is strongly positive with horizontal communication modes and group coordination modes, as predicted by our model. Interestingly, vertical channels show no discernible correlation with task uncertainty.

Table 5 shows the results of a multivariate regression of the different coordination modes against task uncertainty, interdependence, and unit size. The results indicate that task uncertainty decreases impersonal modes of coordination, the rules and instructions provided by the organization, whereas it significantly increases horizontal communication modes as well as group coordination mechanisms. These results confirm that both rules, that is, limited employee discretion, and managerial intervention substitute horizontal communication in the presence of significant uncertainty. ${ }^{31}$

\footnotetext{
${ }^{29}$ The organization of employment security agencies was subject of an extensive and very detailed study in Blau and Schoenherr (1971). Chapter 2 of their book provides an informal description of employment security agencies, whose responsibilities include everything from placement of unemployed workers to counseling and the distribution of unemployed benefits.

${ }^{30}$ Task uncertainty was measured as the average responses to several questions relating to the variability of tasks. For instance, a sample question for task uncertainty was "How much variety in cases, claims, clients, or things do you generally encounter in your normal working day?"

${ }^{31}$ Another interesting study is that of Argote (1982), who investigates the relation between input uncertainty, the means of coordination, and the criteria for organizational effectiveness in a sample of emergency units in several hospitals, which experience a considerable degree of uncertainty on a daily basis such as the overall composition of patient needs and their appropriate treatments. She finds that high input uncertainty correlates
} 


\section{VI.D The impact of task interdependence}

Lincoln, Hanada, and McBride (1986), finally, offer some evidence of the impact of task interdependence on task specialization. Among other things, their study of differences in organizational practices across US and Japanese firms finds that workers in Japanese firms are less specialized. While cultural factors explain this partially, technological factors are important as well. Table 2, which partially reproduces Table 3 in Lincoln et al. (1986), shows the result of a regression of specialization on several variables, of which we report only the significant ones. As can be seen, interdependence increases specialization rather than decrease it, an observation that contradicts the simple trade-off between coordination and specialization emphasized by Becker and Murphy (1992). ${ }^{32}$ In contrast, our model shows how an increase in task interdependence may increase task-specialization as it decreasing employee discretion and, hence, the demand for ex post coordination.

\section{CONCLUSIONS}

In this paper we have argued that endogenizing the demand for coordination is essential in both understanding the problem of organizational design from a theoretical point of view as well as in rationalizing the changes in workplace practices observed over the last two decades. The endogeneity of the demand for coordination induces a profit function for the organization that is often convex in the degree of specialization of its members and in the quality of the horizontal communication between them. The reason is that organizations exhibit increasing returns to coordination, that is, the better the coordination between the members of the organization the higher the demand for further coordination. A direct consequence of this observation is that organizations tend to oscillate between two distinct modes. In the first one, organizations are characterized by very specialized job descriptions and limited employee discretion whereas the second displays broad job classifications and substantial employee discretion. The first, then, coordinates ex-ante, exploiting specialization gains and sacrificing adaptability whereas the second, coordinates ex-post, foregoing the benefits of specialization to gain in adaptability.

The convexity of the profit function with respect to the degree of specialization and the quality of the horizontal communication leads to organizations that are very sensitive to changes in fundamental parameters. A minor change in one of the parameters may lead from one mode of organizational design to another and we have ventured the possibility that this

with "non programmed" means of coordination, such as autonomy of the staff and mutual adjustment, whereas low uncertainty correlates positively with programmed means (use of rules, authority, and scheduled meetings.)

${ }^{32}$ On average, US firms exhibit more workflow rigidity than their Japanese counterparts. 
is the mechanism at work in the recent wave of reengineering and transformations observed in the firms of developed economies.

Importantly, the endogeneity of the demand for coordination offers a more subtle picture of some classic results in the literature. First, specialization is not monotonic in the importance of task interdependence. If task interdependence becomes more important, the organization may abandon adaptability altogether, exploit the specialization gains to the fullest, and ensure coordination by limiting the employee's discretion, that is, by coordinating ex-ante. In this case then, more interdependence leads to more specialization, not less. Second, specialization is not monotonic in improvements in communication technology. In the presence of this improvement it pays to be more adaptive and this increases the demand for coordination. Organizations may meet this additional demand for coordination by decreasing specialization rather than increasing it, and, in this case improvements in communication are accompanied by broader job descriptions and enhanced employee discretion.

Throughout the paper we have maintained a team-theoretic framework in which agents share the same organizational goal. While we believe this is a necessary first step, we are well aware that career concerns and the need to provide individualized incentives may interfere with the objectives of adaptation and coordination. Agents with paternalistic objectives, for example, are unlikely to fully internalize the coordination problems their actions entail for other agents. This may result in over-adaptation. Similarly, if communication is privately costly, agents are likely to underinvest in improving coordination. This moral hazard problem may result in sub-optimal levels of communication. The study of coordination in the presence of incentive conflicts is the topic of our current research. 


\section{REFERENCES}

Aoki, Masahiko (1986), "Horizontal vs. vertical information structure of the firm," American Economic Review, 76, 971-983.

Argote, Linda (1982), "Input Uncertainty and Organizational Coordination in Hospital Emergency Units." Administrative Science Quarterly, 27, 420-434.

Autor, David, Frank Levy, and Richard Murname (2003) "The Skill Content of Recent Technological Change: An Empirical Exploration," Quarterly Journal of Economics, CXVIII, no.4.

Becker, Gary S. and Kevin M. Murphy (1992), "The division of labor, coordination costs, and knowledge," Quarterly Journal of Economics, CVII, 1137-1160.

Blau, Peter M. and Richard A. Schoenherr (1971) The Structure of Organizations, Basic Books, Inc., Publishers, New York.

Bolton, Patrick and Mathias Dewatripont (1994), "The Firm as a Communication Network," Quarterly Journal of Economics, CIX, 809-839.

Boyer, R. (1991) "New Directions in Management Practicesand Work Organization. General Principles and National Trajectories." Couverture Orange. CEPREMAP, no. 9804.

Bresnahan, Timothy F., Erik Brynjolffson, and Lorin M. Hitt (2002) "Information Technology, Workplace Organization, and the Demand for Skilled Labor: Firm-Level Evidence." Quarterly Journal of Economics. 117, 339-376.

Brynjolffson, Erik and Lorin M. Hitt (1998), "Information Technology and Organizational Design: Evidence from Micro Data." Manuscript, MIT.

Bue, J. (1989), "Les Differentes Formes de Flexibilite." Travail et Emploi, 41,

Caroli, Eve (2001) "New Technologies, Organizational Change, and the Skill Bias: What do We Know?" in Pascal Petit and Luc Soete eds. Technology and the Future Employment of Europe. Edward Elgar, London.

Caroli, Eve and John Van Reenen (2001), "Skill Biased Organizational Change? Evidence from a Panel of British and French Establisments." Quarterly Journal of Economics, CXVI, 1449-1492.

Cremer, Jacques (1980), "A partial theory of the optimal organization of a bureaucracy," Bell Journal of Economics, Autumn, 683-693.

Coutrot, T. (1996) "Les Noveaux Modes d'Organisation de la Production: Quels Effets sur l'Emploi, la Formation, l'Organisation du Travail?" Donnees Sociales, INSEE, 209-216.

Crowston, Kevin (1997) "A Coordination Theory Approach to Organizational Process Design," Organization Science, 8, 157-175. 
Curtis, Bill, Herb Krasner, and Neil Iscoe (1988) "A Field Study of the Software Design Process for Large Systems," Communications of the ACM, 31, 1268-1287.

Cyert, R. and D. Mowery (1987) Technology and Employment. Innovation and Growth in the US Economy.National Academy Press. Washington.

Faraj, Samer and Lee Sproull (2000) "Coordinating Expertise in Software Development Teams," Management Science, 46, 1554-1568.

Frechou, H. and N. Greenan (1995) "L'Organisation de la Production dans l'Industrie: des Changements Profondes." 4-pages Sessi.

Garicano, Luis (2000), "Hierarchies and the Organization of Knowledge in Production," Journal of Political Economy, October, 874-904.

Geanakoplos, John and Paul Milgrom (1991), "A theory of Hierachies based on limited managerial attention," Journal of the Japanese and International Economies, 5, 205-225.

Holmstrom, Bengt and Paul Milgrom (1994), "The Firm as an Incentive System," American Economic Review, 84, 972-991

Hammer, Michael and James Champy (2001) Reengineering the Corporation, Harper Business, New York, 2nd ed.

Kraut, Robert E. and Lynn A. Streeter (1995), "Coordination in Software Development," Communications of the ACM, 38, 69-81.

Lincoln, James R., Mitsuyo Hanada, and Kerry mcBride (1986), "Organizational Structure in Japanes and US Manufacturing." Administrative Science Quarterly, 31, 338-364.

Lindbeck, Assar and Dennis J. Snower (2000), "Multitask learning and the reorganization of wok: From tayloristic to holistic organization," Journal of Labor Economics, 18, 353-376.

March, J. and H. Simon (1958) Organizations. Wiley. New York.

Marschak, Jacob and Roy Radner (1972), Economic Theory of Teams, Yale University Press, New Haven and London.

Maurin, Eric and David Thesmar (2003) "Change in the Functional Structure of Firms and the Demand for Skill." Journal of Labor Economics, forthcoming.

Milgrom, Paul and John Roberts (1990), "The economics of modern manufacturing: Technology, strategy, and organization," American Economic Review, 80, 511-528.

Milgrom, Paul and John Roberts (1992), Economics, Organization, and Management, Prentice Hall, Englewood Cliffs, New Jesey.

Milgrom, Paul and John Roberts (1995), "Complementarities and fit. Strategy, structure, and organizational change in manufacturing." Journal of Accounting and Economics, 19, 179-208. 
OECD (1999) Employment Outlook. OECD. Paris.

Osterman, Paul (1994), "How Common is Workplace Transformation and Who Adopts It?" Industrial and Labor Relations Review, 47, 173-188.

Radner, Roy (1993), "The Organization of Decentralized Information Processing," Econometrica, 61, 1109-1146.

Radner, Roy and Timothy Van Zandt (1992), "Information Processing in Firms and Returns to Scale," Annales D'Economie Et De Statistique, 25-26, 265-298.

Rajan, Raghuram and Julie Wulf (2003) "The Flattening Firm: Evidence from Panel Dat on the Changing Nature of Corporate Hierarchies." Manuscript. Graduate School of Business. University of Chicago.

Smith, Adam (1981) An Inquiry into the Nature and Causes of the Wealth of Nations. Liberty Funds. Indianopolis.

Van de Ven, Andrew H, Andre L. Delbecq, and Richard Koenig, Jr. (1976), "Determinants of Coordination Modes within Organizations," American Sociological Review, 41, 322-338.

Van Zandt, Timothy (1999), "Real-Time Decentralized Information Processing as a Model of Organizatsions with Boundedly Rational Agents," Review of Economic Studies, 66, 633-658.

Vayanos, Dimitri (2003) "The Decentralization of Informatioin Processing in the Presence of Interactions." Review of Economic Studies. 70, 667-695.

Zmud, Robert W. (1980) "Management of Large Software Development Efforts," MIS Quarterly, $4,45-55$. 


\section{APPENDIX}

Proof of Lemma 1: Clearly the choice of $a^{j i}$ for $j \in \mathcal{T}(i), j \neq i$ or $j \notin \mathcal{T}(i)$ when the agent in charge of task $j$ observes $a^{i i}$ is $a^{j i}=a^{i i}$. When instead the agent in charge of task $j \notin T(i)$ does not observe $a^{i i}$, the agent minimizes $E\left[\beta\left(a^{j i}-a^{i i}\right)^{2}\right]$ and the solution is $a^{j i}=E\left(a^{i i}\right)$. The choice of $a^{i i}$ then follows from the the minimization of the the function:

$$
\min _{a^{i i}}\left\{\phi\left(a^{i i}-\theta^{i}\right)^{2}+\beta(1-p)(n-t)\left(E\left(a^{i i}\right)-a^{i i}\right)^{2}\right\},
$$

and the solution is given by

$$
a^{i i}=\left(\frac{\phi}{\phi+B}\right) \theta^{i}+\left(\frac{B}{\phi+B}\right) E\left(a^{i i}\right)
$$

where $B=\beta(n-t)(1-p)$. Clearly then $E\left(a^{i i}\right)=\widehat{\theta}^{i}$.

Proof of Lemma 2: Substitution of $(A 2)$ in $(A 1)$ together with the fact that $a^{j i}=E\left(a^{i i}\right)=\widehat{\theta}^{i}$ for $j \notin T(i)$ when the agent in charge of task $j$ does not observe $a^{i i}$ yields

$$
\phi\left(\frac{B}{\phi+B}\right)\left(\theta^{i}-\widehat{\theta}^{i}\right)^{2} .
$$

Adding up over $i$, substracting $h(t, \alpha)$ and $\delta g(p)$ and adding up over $i$ yields $\Pi(p, t)$.

\section{Proof of Proposition 3}

\section{Preliminaries}

Define

$$
\pi(\widehat{p}, t)=\max _{p \geq \widehat{p}} \Pi(p, t)
$$

The choice of $\widehat{p}$ then does not affect the optimal choice of the other design variables as, given the strict quasiconcavity of the profit function, the highest optimal value of $\widehat{p}$ is always chosen to be equal to $p^{*}(t)$, the unique equilibrium value for the quality of communication. With some abuse of notation, define $\widetilde{p}(t)$ as the value of the constrained maximization of the profit function,

$$
\widetilde{p}(t)=\left\{\begin{array}{ccc}
p^{*}(t) & \text { if } & p^{*}(t)>\widehat{p} \\
\widehat{p} & \text { if } & p^{*}(t) \leq \widehat{p}
\end{array} .\right.
$$

Recall as well that we have assumed that $\Pi(p, t)$ is a strictly quasiconcave function of $p$. $\Pi(p, t, \tau)$ is a differentiable function of $p$ and, as a consequence, it follows that $p^{*}(t)$ is the solution of $\Pi_{p}(p, t)=0$, where

$$
\Pi_{p}(p, t)=-\delta n(n-t) g_{p}(p)-n\left(\frac{\phi}{\phi+B(p, t)}\right)^{2} B_{p}(t) \sigma_{\theta}^{2},
$$

where $B_{p}(t)=-\beta(n-t)$. In addition, recall that we have assumed that

$$
g_{p}(0)=0 \text { and } \quad \lim _{p \rightarrow 1} g_{p}(p)=\infty .
$$

It follows that $p^{*}(t)$ is unique, strictly in the interior of $[0,1]$, and differentiable with respect to any of the parameters $\tau \in\left\{\sigma_{\theta}^{2}, \phi,-\alpha\right\}$. Finally notice that, 


$$
B_{\widehat{p}}(t)=\left\{\begin{array}{ccc}
0 & \text { if } & p^{*}(t)>\widehat{p} \\
-\beta(n-t) & \text { if } & p^{*}(t) \leq \widehat{p}
\end{array} \quad \leq 0,\right.
$$

The following lemma plays an important role in what follows.

Lemma I $\widetilde{p}(\bar{t}) \geq \widetilde{p}(\underline{t})$ for $\bar{t}>\underline{t}$ and $\bar{t}, \underline{t} \in \mathcal{J}$

Proof: Clearly it is enough to show that $p^{*}(\bar{t})>p^{*}(\underline{t})$. Then

$$
\begin{aligned}
0 & =\Pi_{p}\left(p^{*}(\underline{t}), \underline{t}\right) \\
& =(n-\underline{t})\left\{-\delta n g_{p}\left(p^{*}(\underline{t})\right)+n \beta\left(\frac{\phi}{\phi+B\left(p^{*}(\underline{t}), \underline{t}\right)}\right)^{2} \sigma_{\theta}^{2}\right\} \\
& <(n-\underline{t})\left\{-\delta n g_{p}\left(p^{*}(\underline{t})\right)+n \beta\left(\frac{\phi}{\phi+B\left(p^{*}(\underline{t}), \bar{t}\right)}\right)^{2} \sigma_{\theta}^{2}\right\} \\
& =\left(\frac{n-\underline{t}}{n-\bar{t}}\right)\left\{-\delta n(n-\bar{t}) g_{p}\left(p^{*}(\underline{t})\right)+n \beta(n-\bar{t})\left(\frac{\phi}{\phi+\frac{1}{x^{m}}+B\left(p^{*}(\underline{t}), \bar{t}\right)}\right)^{2}\right\} \\
& =\left(\frac{n-\underline{t}}{n-\bar{t}}\right) \Pi_{p}\left(p^{*}(\underline{t}), \bar{t}\right),
\end{aligned}
$$

where the strict inequality follows from the fact that $B\left(p^{*}(\underline{t}), \bar{t}\right)<B\left(p^{*}(\underline{t}), \underline{t}\right)$. The result now follows from the strict quasiconcavity of $\Pi(p, t, \tau)$.

Proof of Proposition 3

(i) To prove this part of the proposition we make use of standard results on supermodularity. By Theorem 1 of Milgrom and Roberts (1990), ${ }^{33}$ a function $\ell: \mathbb{R}^{m} \rightarrow \mathbb{R}$ that is supermodular when exclusively considered as a function of two variables $y^{i}$ and $y^{j}$ while fixing the remaining ones, $\ell\left(y^{i}, y^{j}, y^{-i j}\right)$, is supermodular. Our strategy of proof is then to show that the cross derivative of the function $\pi(\widehat{p}, t, \tau)$ are all positive, where we have added the parameter $\tau \in\left\{\sigma_{\theta}^{2}, \phi,-\alpha\right\}$ as an additional argument in the function. Throughout we appeal to the envelope theorem and "ignore" the impact of the variation on, say, $\tau$ on $p^{*}(t, \tau)$ whenever $p^{*}(t, \tau)>\widehat{p}$. Also notice that whenever $p^{*}(t, \tau) \leq \widehat{p}$ the constrained maximization of $p$ sets $\widetilde{p}(t, \tau)=\widehat{p}$ and hence, for example,

$$
\widetilde{p}_{\tau}=\left\{\begin{array}{ccc}
p_{\tau}^{*} & \text { if } & p^{*}(t, \tau)>\widehat{p} \\
0 & \text { if } & p^{*}(t, \tau) \leq \widehat{p}
\end{array}\right.
$$

We prove the properties with respect to $\phi$, the proof with respect to $-\alpha$ and $\sigma_{\theta}^{2}$ being identical in its logic.

Complementarity between $\widehat{p}$ and $t$ : Define

\footnotetext{
${ }^{33}$ Milgrom and Roberts (1995) offer a transparent primer on the mathematics of supermodularity. For a textbook presentation see Sundaram (1996).
} 


$$
\Delta(\widehat{p})=\pi(\widehat{p}, \bar{t}, \phi)-\pi(\widehat{p}, \underline{t}, \phi) \quad \text { for } \quad \bar{t}>\underline{t} \quad \text { and } \quad \bar{t}, \underline{t} \in \mathcal{J}
$$

Notice that if $\widetilde{p}(\bar{t})>\widetilde{p}(\underline{t}) \geq \widehat{p}$, then, trivially $\Delta_{\widehat{p}}=0$. If instead, $\widetilde{p}(\bar{t})>\widehat{p}>\widetilde{p}(\underline{t})$, then $\Delta_{\widehat{p}}=$ $-\pi_{\widehat{p}}(\widehat{p}, \underline{t}, \phi)>0$, by the strict quasiconcavity of the profit function and the fact that $\widehat{p}>\widetilde{p}(\underline{t})$. Asume finally that $\widehat{p} \geq \widetilde{p}(\bar{t})>\widetilde{p}(\underline{t})$. In this case,

$$
\begin{aligned}
\Delta_{\widehat{p}} & =\delta n(\bar{t}-\underline{t}) g_{p}(\widehat{p})+n \phi^{2} \sigma_{\theta}^{2}\left\{\frac{-B_{\widehat{p}}(\bar{t})}{(\phi+B(\widehat{p}, \bar{t}))^{2}}-\frac{-B_{\widehat{p}}(\underline{t})}{(\phi+B(\widehat{p}, \underline{t}))^{2}}\right\} \\
& >\delta n(\bar{t}-\underline{t}) g_{p}(\widehat{p})+n\left(\frac{\phi}{\phi+B(\widehat{p}, \bar{t})}\right)^{2} \sigma_{\theta}^{2}\left(-B_{\widehat{p}}(\bar{t})+B_{\widehat{p}(\underline{t}))}\right. \\
& =(\bar{t}-\underline{t})\left\{\delta n g_{p}(\widehat{p})-\beta n\left(\frac{\phi}{\phi+B(\widehat{p}, \bar{t})}\right)^{2} \sigma_{\theta}^{2}\right\} \\
& =\left(\frac{\bar{t}-\underline{t}}{n-\bar{t}}\right)\left\{\delta n(n-\bar{t}) g_{p}(\widehat{p})-\beta n(n-\bar{t})\left(\frac{\phi}{\phi+B(\widehat{p}, \bar{t})}\right)^{2} \sigma_{\theta}^{2}\right\} \\
& =\left(\frac{\bar{t}-\underline{t}}{n-\bar{t}}\right)\left\{\delta n(n-\bar{t}) g_{p}(\widehat{p})+n\left(\frac{\phi}{\phi+B(\widehat{p}, \bar{t})}\right)^{2} B_{\widehat{p}}(\bar{t}) \sigma_{\theta}^{2}\right\} \\
& =-\pi_{\widehat{p}}(\widehat{p}, \bar{t}, \phi) \\
& >0 .
\end{aligned}
$$

Complementarity between $\widehat{p}$ and $\phi:$ In this case,

$$
\pi_{\widehat{p} \phi}=-2 n \phi \sigma_{\theta}^{2} B_{\widehat{p}}\left[\frac{B-\phi B_{\widetilde{p}} \widetilde{p}_{\phi}}{(\phi+B)^{3}}\right] \geq 0,
$$

where $\widetilde{p}_{\phi}>0$ if $p^{*}(t, \tau)>\widehat{p}$, as it follows from applying the implicit function theorem to $\Pi_{p}(p, t)=0$, and $\widetilde{p}_{\phi}=0$ if $p^{*}(t, \tau) \leq \widehat{p}$.

Complementarity between $t$ and $\phi:$ Define

$$
\Delta(\phi)=\pi(\widehat{p}, \bar{t}, \phi)-\pi(\widehat{p}, \underline{t}, \phi)
$$

Then

$$
\Delta_{\phi}=n\left\{\left(\frac{B(\widetilde{p}(\underline{t}), \underline{t})}{\phi+B(\widetilde{p}(\underline{t}), \underline{t})}\right)^{2}-\left(\frac{B(\widetilde{p}(\bar{t}), \bar{t})}{\phi+B(\widetilde{p}(\bar{t}), \bar{t})}\right)^{2}\right\} \sigma_{\theta}^{2}>0
$$

as $B(\widetilde{p}(\bar{t}), \bar{t})<B(\widetilde{p}(\underline{t}), \underline{t})$.

(ii) This follows immediately from Lemma I and $(i)$ above.

(iii) Denote $t^{1}=t^{*}\left(\beta^{1}\right)$ and $t^{2}=t^{*}\left(\beta^{2}\right)$ with $\beta^{1} \neq \beta^{2}$. Assume that $t^{2}>t^{1}$. Let $\widetilde{p}^{1}(t)$ denote the optimized value of $p$ given $\beta^{1}$ and $t \in \mathcal{J}$, and let $\widetilde{p}^{2}(t)$ denote the optimized value of $p$ given $\beta^{2}$ and $t \in \mathcal{J}$. From Lemma I, it follows that $\widetilde{p}^{2}\left(t^{2}\right)>\widetilde{p}^{2}\left(t^{1}\right)$. In addition, from A1, for any $\delta>0$ there 
exists an $\varepsilon>0$ such that whenever $\left|\beta^{2}-\beta^{1}\right|<\varepsilon$, then $\left|\widetilde{p}^{2}(t)-\widetilde{p}^{1}(t)\right|<\delta$. It follows that there exists an $\varepsilon>0$ such that whenever $\left|\beta^{2}-\beta^{1}\right|<\varepsilon, p^{*}\left(\beta^{2}\right)=\widetilde{p}^{2}\left(t^{2}\right)>\widetilde{p}^{1}\left(t^{1}\right)=p^{*}\left(\beta^{1}\right)$.

Proof of Proposition 4: As $\beta \longrightarrow \infty$ the organization will find it necessary to achieve perfect coordination in equilibrium, that is for all $i, j a^{j i}=a^{i i}$. Given that $p<1$ and $t<n$, this implies that $a^{j i}=a^{i i}=\hat{\theta}^{i i}$. However, whenever $a^{j i}=a^{i i}=\hat{\theta}^{i i}$ for all $i, j$, positive returns to specialization imply that it will be optimal to choose $t=1$. Hence, as $\beta \longrightarrow \infty$ either $t=\hat{t}$ or $t=1$. Obviously, if $\phi$ tends to $0, t^{*}=1$ will be optimal, whereas $t^{*}=\hat{t}$ will be optimal whenever $\phi$ tends to $\infty$. Moreover, if $t^{*}=\hat{t}$ for $\phi^{\prime}$ then also $t^{*}=\hat{t}$ for $\phi>\phi^{\prime}$. This proves part (a) of the proposition. Finally, if given $\alpha=\alpha^{\prime}$ for $\phi=\phi^{\prime}$, then the organization is indifferent between setting $t^{*}=1$ and setting $t^{*}=\hat{t}$ then for $\alpha>\alpha^{\prime}$ the organization must strictly prefer $t=1$ which implies the second part of the proposition.

Proof of Proposition 6: We have that $\lim _{\delta \longrightarrow \infty} p^{*}=0$, where $p^{*}$ is the equilibrium level of communication regardless of the value of $t$. Since

$$
\Pi(p, t) \equiv-n \phi\left(\frac{B}{\phi+B}\right) \sigma_{\theta}^{2}-n(n-t) \delta g(p)-n h(t, \alpha)
$$

it is easy to show that then also

$$
\lim _{\delta \longrightarrow \infty} \delta g(p)=0
$$

This implies that

$$
t=\arg \max _{t}\left\{-n \phi\left(\frac{\beta(n-t)}{\phi+\beta(n-t)}\right) \sigma_{\theta}^{2}-n h(t, \alpha)\right\}
$$

Since the first term between parenthesis is both strictyly increasing in $\phi$ and in $t$, it follows that there exists a $\phi^{\prime}$ such that the solution equals 1 if and only if $\phi \leq \phi^{\prime}$. If an increase in $\alpha$ increases $h(t, \alpha)$ for all values of $t$ except $t=1$, then $\bar{\phi}$ is increasing in $\alpha$.

Proof of Lemma 8: $(a)$ Let $E\left(C^{i} \mid \varepsilon^{i}\right.$, int $)$ the expected cost function associated with task $i$, conditional on the management's information at the interim stage, that is conditional on $\varepsilon^{i}$, when management decides to intervene and update the rules. Similarly let $E\left(C^{i} \mid \varepsilon^{i}\right.$, non-int $)$ the corresponding expected cost function conditional on the managemet's information at the interim stage when management decides not to intervene. Clearly, in the case of intervention, $E\left(C^{i} \mid \varepsilon^{i}\right.$,int $)$ is given as in Lemma 3 ,

$$
E\left(C^{i} \mid \varepsilon^{i}, \text { int }\right)=\phi\left(\frac{B}{\phi+B}\right) \sigma_{\theta}^{2}+\omega m+I(m)+\delta(n-t) g(p)
$$

where the term $I(m)$ is the cost associated with intervention. If management decides not to intervene and update the rules associated with task $i$, the organization saves managerial intervention costs $I(m)$, but suffers the costs associated with the bias built in the outdated rules,

$$
E\left(C^{i} \mid \varepsilon^{i}, \text { non-int }\right)=E\left(C^{i} \mid \varepsilon^{i}, \text { int }\right)+\phi\left(\frac{B}{\phi+B}\right) \varepsilon^{i^{2}}-I(m)
$$


Mangement then intervenes in task $i$, whenever $E\left(C^{i} \mid \varepsilon^{i}\right.$, non-int $)>E\left(C^{i} \mid \varepsilon^{i}\right.$, int $)$, that is, management intervenes whenever,

$$
\left|\varepsilon^{i}\right|>b \quad \text { where } \quad b=\left[\left(\frac{\phi+B}{B}\right) \frac{I(m)}{\phi}\right]^{\frac{1}{2}}
$$

which concludes the proof of Lemma 8. (b) This follows immediately from the expression for $b$.

Proof of Lemma 9: Recall that $P^{i}=\operatorname{prob}\left[\left|\varepsilon^{i}\right| \geq b\right]$, then

$$
\begin{aligned}
E\left(C^{i}\right) & =(1-P) E\left(C^{i}|| \varepsilon^{i} \mid<b\right)+P E\left(C^{i}|| \varepsilon^{i} \mid \geq b\right) \\
& =\delta(n-t) g(p)+\omega m \\
& +(1-P) \phi\left(\frac{B}{\phi+B}\right)\left(\sigma_{\theta}^{2}+E\left[\varepsilon^{i^{2}}|| \varepsilon^{i} \mid<b\right]\right) \\
& +P\left[\phi\left(\frac{B}{\phi+B}\right) \sigma_{\theta}^{2}+I(m)\right],
\end{aligned}
$$

adding and substracting

$$
P \phi\left(\frac{B}{\phi+B}\right) E\left[\varepsilon^{i^{2}}|| \varepsilon^{i} \mid \geq b\right]
$$

the results follows.

\section{Proof of Proposition 10}

Preliminaries

Recall that the second period profit function is given by,

$$
\pi(\widehat{p}, t, b,-m, \tau)=\max _{p \geq \widehat{p}} \Pi(p, t, b,-m),
$$

for $\tau \in\left\{\sigma_{\theta}^{2},-\alpha, \omega\right\}$. The following Lemma, which is given without proof, is the counterpart to Lemma I above and we use it repeatedly in the proof of Proposition 10.

Lemma II (a) $\widetilde{p}(\bar{t}) \geq \widetilde{p}(\underline{t})$ for $\bar{t}>\underline{t}$ and (b) $\widetilde{p}_{b}>0$.

\section{Proof of Proposition 10}

To show (i) and (ii) it is enough to prove that, say,

$$
\pi(\widehat{p}, t, b,-m,-\alpha),
$$

is supermodular, the proof with respect to $\sigma_{\theta}^{2}$ and $\omega$ being identical in the logic and mechanics. Importantly in what follows, it is easy to check that the optimal level of the quality of the communication, $p^{*}$ is independent of $m$, the size of the managerial force, and $-\alpha$.

Complementarity between $\widehat{p}$ and $t$ : Define

$$
\Delta(\widehat{p})=\pi(\widehat{p}, \bar{t}, b,-m,-\alpha)-\pi(\widehat{p}, \underline{t}, b,-m,-\alpha) .
$$

The proof that $\Delta_{\widehat{p}}>0$ follows identical steps as those in Proposition 3 , with the only substitution of $\sigma_{\theta}^{2}$ by

$$
\sigma_{\theta}^{2}+(1-P) E\left[\varepsilon^{i^{2}}|| \varepsilon^{i} \mid<b\right] .
$$


Complementarity between $\widehat{p}$ and $b$ : In this case

$$
\pi_{\widehat{p} b}=-n B_{\widehat{p}}\left(\frac{\phi}{\phi+B}\right)^{2}\left\{-P_{b} E\left[\varepsilon^{i^{2}}|| \varepsilon^{i} \mid<b\right]+(1-P) E_{b}\left[\varepsilon^{i^{2}}|| \varepsilon^{i} \mid<b\right]\right\}>0 .^{34}
$$

Complementarity between $\widehat{p}$ and $-m: \quad \pi_{\widehat{p}(-m)}=0$.

Complementarity between $\widehat{p}$ and $-\alpha: \quad \pi_{\widehat{p}(-\alpha)}=0$.

Complementarity between $t$ and $b$ : Define

$$
\Delta(b)=\pi(\widehat{p}, \bar{t}, b,-m,-\alpha)-\pi(\widehat{p}, \underline{t}, b,-m,-\alpha) .
$$

Then

$$
\begin{aligned}
\Delta_{b} & =-\left[\frac{B(\widetilde{p}(\bar{t}), \bar{t})}{\phi+B(\widetilde{p}(\bar{t}), \bar{t})}-\frac{B(\widetilde{p}(\underline{t}), \underline{t})}{\phi+B(\widetilde{p}(\underline{t}), \underline{t})}\right] \\
& \times\left\{-P_{b} E\left[\varepsilon^{i^{2}}|| \varepsilon^{i} \mid<b\right]+(1-P) E_{b}\left[\varepsilon^{i^{2}}|| \varepsilon^{i} \mid<b\right]\right\} \\
& >0
\end{aligned}
$$

where we have made use of the enevelope theorem to ignore the effect of variation in $b$ in $\widetilde{p}$. Complementarity between $t$ and $-m$ : Define

$$
\Delta(-m)=\pi(\widehat{p}, \bar{t}, b,-m,-\alpha)-\pi(\widehat{p}, \underline{t}, b,-m,-\alpha) .
$$

Then

$$
\Delta_{(-m)}=0 .
$$

Complementarity between $t$ and $-\alpha$ : Define

$$
\Delta(-\alpha)=\pi(\widehat{p}, \bar{t}, b,-m,-\alpha)-\pi(\widehat{p}, \underline{t}, b,-m,-\alpha) .
$$

Then

$$
\Delta_{(-\alpha)}=h(\bar{t}, \alpha)-h(\underline{t}, \alpha)>0
$$

as, by our assumptions, $h_{t \alpha}>0$.

Complementarity between $b$ and $-m: \quad \pi_{b(-m)}=P_{b} I_{m}>0$, as recall that $h_{m}<0$.

Complementarity between $b$ and $-\alpha: \quad \pi_{b(-\alpha)}=0$.

Complementarity between $-m$ and $-\alpha: \quad \pi_{(-m)(-\alpha)}=0$.

This concludes the proof of Proposition 10.

\footnotetext{
${ }^{34}$ Clearly $B_{\widehat{p}} B_{\widetilde{p}}=0$ so the term in $B_{\widetilde{p}}$ can be ignored.
} 


\section{Proof of Proposition 11}

Preliminaries

Start by defining,

$$
\pi(\widehat{p}, t, x, \tau)=\max _{p \geq \widehat{p}} \Pi(p, t, x, \tau)
$$

for $\tau \in\left\{\sigma_{\theta}^{2}, \phi,-\alpha,-\lambda\right\}$.Define,

$$
\widetilde{p}(t, x)=\left\{\begin{array}{ccc}
p^{*}(t, x) & \text { if } & p^{*}(t, x)>\widehat{p} \\
\widehat{p} & \text { if } & p^{*}(t, x) \leq \widehat{p}
\end{array},\right.
$$

where $p^{*}(t, x)$ is defined as the solution to the first order condition

$$
0=-\delta n(n-t) g_{p}(p)-n q(x)\left(\frac{\phi}{\phi+B(p, t)}\right)^{2} B_{p}(t) \sigma_{\theta}^{2}
$$

Then we prove the following Lemma.

Lemma III (a) $\widetilde{p}(\bar{t}, x) \geq \widetilde{p}(\underline{t}, x)$ for $\bar{t}>\underline{t}, \bar{t}, \underline{t} \in \mathcal{J}$ and (b) $\widetilde{p}(t, \bar{x}) \geq \widetilde{p}(t, \underline{x})>0$ for $\bar{x}>\underline{x}$.

Proof: The proof of part (a) is identical to that of Lemma I. As for (b), it is enough to prove the result for $p^{*}(t, x)$. Let $\underline{p}=p^{*}(t, \underline{x})$. Then the first order condition when $x=\underline{x}$ is

$$
\begin{aligned}
0 & =-\delta n(n-t) g_{p}(\underline{p})-n q(\underline{x})\left(\frac{\phi}{\phi+\beta(n-t)(1-\underline{p})}\right)^{2} B_{p} \sigma_{\theta}^{2} \\
& <-\delta n(n-t) g_{p}(\underline{p})-n q(\bar{x})\left(\frac{\phi}{\phi+\beta(n-t)(1-\underline{p})}\right)^{2} B_{p} \sigma_{\theta}^{2} \\
& =\Pi_{p}(\underline{p}, t, \bar{x}, \tau)
\end{aligned}
$$

and thus $p^{*}(t, \bar{x})>\underline{p}=p^{*}(t, \underline{x})$.

The logic of the proof of Proposition 11 is identical to that of Proposition 3. As before as well we appeal to the envelope theorem and "ignore" the impact of the variation on, say, $\tau$ on $p^{*}(t, \tau)$ whenever $p^{*}(t, \tau)>\widehat{p}$.

\section{Proof of Proposition 11}

(i) We prove the result for the case where $\tau=-\lambda$. The proof for the rest of the parameters is identical. Complementarity between $\widehat{p}$ and $t$ : Define

$$
\Delta(\widehat{p})=\pi(\widehat{p}, \bar{t}, x,-\lambda)-\pi(\widehat{p}, \underline{t}, x,-\lambda) \quad \text { for } \quad \bar{t}>\underline{t} \quad \text { and } \quad \bar{t}, \underline{t} \in \mathcal{J}
$$

That $\Delta_{\widehat{p}} \geq 0$ then is identical to the case of Proposition 3 .

Complementarity between $\widehat{p}$ and $x$ : Define

$$
\Delta(\widehat{p})=\pi(\widehat{p}, t, \bar{x},-\lambda)-\pi(\widehat{p}, t, \underline{x},-\lambda) \quad \text { for } \quad \bar{x}>\underline{x}
$$


Notice that if $\widetilde{p}(t, \bar{x})>\widetilde{p}(t, \underline{x}) \geq \widehat{p}$, then, trivially $\Delta_{\widehat{p}}=0$. Next if, by Lemma III, $\widetilde{p}(t, \bar{x}) \geq \widehat{p}>\widetilde{p}(t, \underline{x})$, then

$$
\Delta_{\widehat{p}}=\delta n(n-t) g_{p}(\widehat{p})+n q(\underline{x})\left(\frac{\phi}{\phi+\beta(n-t)(1-\widehat{p})}\right)^{2} B_{\widehat{p}} \sigma_{\theta}^{2}=-\Pi_{p}(\widehat{p}, t, \underline{x}, \tau)>0,
$$

by the strict quasiconcavity $\Pi(p, t, x, \tau)$ and $\widehat{p}>\widetilde{p}(t, \underline{x})$. Finally, if $\widehat{p} \geq \widetilde{p}(t, \bar{x})>\widetilde{p}(t, \underline{x})$ then

$$
\Delta_{\widehat{p}}=-n(q(\bar{x})-q(\underline{x}))\left(\frac{\phi}{\phi+B(\widehat{p})}\right)^{2} B_{\widehat{p}} \sigma_{\theta}^{2}>0,
$$

as $B_{\widehat{p}}<0$ and $q(\bar{x})>q(\underline{x})$.

Complementarity between $\widehat{p}$ and $-\lambda: \quad \pi_{\widehat{p}(-\lambda)}=0$.

Complementarity between $t$ and $x$ : Define

$$
\Delta(x)=\pi(\widehat{p}, \bar{t}, x,-\lambda)-\pi(\widehat{p}, \underline{t}, x,-\lambda) \quad \text { for } \quad \bar{t}>\underline{t} \quad \text { and } \quad \bar{t}, \underline{t} \in \mathcal{J}
$$

Then if $\widetilde{p}(\bar{t}, x)>\widetilde{p}(\underline{t}, x) \geq \widehat{p}$

$$
\Delta_{x}=n q_{x}\left\{\frac{\phi^{2}}{\phi+B(\widetilde{p}(\bar{t}, x), \bar{t})}-\frac{\phi^{2}}{\phi+B(\widetilde{p}(\underline{t}, x), \underline{t})}\right\}>0,
$$

as $q_{x} \geq 0$ and $B(\widetilde{p}(\bar{t}, x), \bar{t})<B(\widetilde{p}(\underline{t}, x), \underline{t})$.

Complementarity between $t$ and $-\lambda$ : Define

$$
\Delta(-\lambda)=\pi(\widehat{p}, \bar{t}, x,-\lambda)-\pi(\widehat{p}, \underline{t}, x,-\lambda) \quad \text { for } \quad \bar{t}>\underline{t} \quad \text { and } \quad \bar{t}, \underline{t} \in \mathcal{J}
$$

Then clearly,

$$
\Delta_{(-\lambda)}=0 .
$$

Complementarity between $x$ and $-\lambda: \quad \pi_{x(-\lambda)}=C_{x \lambda}>0$.

(ii) This follows immediately from (i) and the fact that $q_{x}>0$. 
Table 1: Organizational change in Britain

(Source: Caroli and Van Reenen (2001))

Have more or less responsibility

\begin{tabular}{cccccccc}
\hline \hline \multicolumn{4}{c}{ Non-Manual } & \multicolumn{4}{c}{ Manual } \\
More & Same & Less & $\mathrm{D} / \mathrm{K}$ & More & Same & Less & $\mathrm{D} / \mathrm{K}$ \\
.462 & .465 & .029 & .045 & .328 & .571 & .064 & .036 \\
\hline
\end{tabular}

Effect on range of tasks performed

\begin{tabular}{llllllll}
\hline \hline \multicolumn{4}{c}{ Non-Manual } & \multicolumn{4}{c}{ Manual } \\
Wider & Same & Narrower & $\mathrm{D} / \mathrm{K}$ & Wider & Same & Narrower & $\mathrm{D} / \mathrm{K}$ \\
.625 & .281 & .058 & .030 & .395 & .450 & .133 & .020 \\
\hline
\end{tabular}

Notes to Table 1: Answers given by senior management to questions concerning organizational change asked in the 1984 WIRS. The questions were asked only if some organizational change had taken place in the last three years. For instance, 46.2 of senior managers responded that workers had more responsibility following organizational change. D/K: don't know or not answered 
Table 2: IT and the division of labor

(Source Brynjolffson and Hitt (1998))

\begin{tabular}{ccccccc}
\hline \hline Variable & IT Capital & MIPS & TOTPC & \% Comp. & \% Email & Comp. \\
Self man. teams & $.17^{* * *}$ & $.22^{* * *}$ & $.20^{* * *}$ & $.17^{* * *}$ & $.27^{* * *}$ & $.18^{* * *}$ \\
Broad jobs & .07 & $.12^{* * *}$ & $.10^{* * *}$ & $.20^{* * *}$ & $.17^{* * *}$ & $.24^{* * *}$ \\
\hline
\end{tabular}

Notes to Table 2: Correlation coefficients. Data from Computer Intelligence. IT Capital: Total purchase value of computer equipment (mainframes, minicomputers and peripherals) during the period 1991-1994. MIPS: Total processing power of central processors, measured in millions of instructions per second. TOTPC: Total number of personal computers in use at the firm. Computer Intelligence Inforcorp database details IT spending by site for companies in the Fortune 1000 companies. Survey questions: \% Comp (\% Email) is the answer to "What fraction of your employees uses general purpose computer (E-mail) in the course of their regular work?" in the survey conducted by Brynjolffson and Hitt (1998). Self man. teams: "Would you say your firm uses self-managing teams very heavily, heavily, moderately, or slightly?" Broad jobs: "How would you rate the amount of diversity associated with production work itself, very high, moderately high, medium, moderately low, or very low?" *** denotes significant at the $1 \%$ level.

Table 3: Specialization and task interdependence in the US and Japan (Source: Lincoln, Hanada, and McBride (1986))

\begin{tabular}{|c|c|}
\hline \hline & Specialization \\
\hline Ind. Variables & \\
US/Japan dummy & $.455^{* * *}$ \\
Ind. Company & $.074^{*}$ \\
Size & $.104^{* * *}$ \\
Task interdependence & $.164^{*}$ \\
$R^{2}$ & $54.2 \%$ \\
\hline
\end{tabular}

Notes to Table 3: Regression of the Aston specialization scale (see Pugh et al. (1968)) on plant attributes. US/Japan dummy takes the value 1 if the establishment is in the US. Ind. Company is a dummy variable taking the value 1 if the plant is a stand alone and 0 if it is a branch plant or subsidiary. Size denotes the number of employees in the plant. Task interdependence is the Aston workflow rigidity scale and characterize both rigidity and task interdependence. ${ }^{*}$ denotes significance at the $10 \%$ and ${ }^{* * *}$ denotes significance at the $1 \%$ level. 
Table 4: Zero order correlation among independent variables and coordination modes (Source: van de Ven, Delbeq, and Koenig (1976))

\begin{tabular}{l|ccccccccccc}
\hline & 1 & 2 & 3 & 4 & 5 & 6 & 7 & 8 & 9 & 10 & 11 \\
\hline \hline 1. Task Uncertainty & & & & & & & & & & & \\
2. Task interdependence & .37 & & & & & & & & & \\
3. Unit size & -.14 & -.12 & & & & & & & & & \\
4. Impersonal coordination & -.49 & -.26 & .29 & & & & & & & & \\
5. Rules and procedures & -.46 & -.22 & .16 & .78 & & & & & & & \\
6. Plans and schedules & -.36 & -.23 & .25 & .75 & .49 & & & & & & \\
7. Personal coordination & .35 & .20 & .03 & .00 & -.05 & -.04 & & & & & \\
8. Vertical channels & .04 & .06 & .11 & .31 & .27 & .23 & .74 & & & & \\
9. Horizontal channels & .52 & .23 & -.06 & -.30 & -.34 & -.28 & .75 & .13 & & & \\
10. Group coordination & .64 & .41 & -.15 & -.32 & -.32 & -.26 & .42 & .11 & .52 & & \\
11. Scheduled meetings & .59 & .41 & -.08 & -.27 & -.27 & -.22 & .36 & .17 & .37 & .88 & \\
12. Unscheduled meetings & .64 & .32 & -.16 & -.33 & -.33 & -.28 & .35 & .02 & .51 & .89 & .66 \\
\hline \hline
\end{tabular}

Table 5: Multiple regression analysis

(Source: Van de Ven, Delbeq, and Koenig (1976))

\begin{tabular}{l|cccc}
\hline Dependent Variable & Task uncertainty & Task interdependence & Unit size & $R^{2}(\%)$ \\
\hline \hline A. Impersonal mode & $-.44(.06)$ & $-.07(.06)$ & $.22(.06)$ & 30 \\
1. Rules and Proc. & $-.43(.07)$ & $-.05(.07)$ & $.10(.06)$ & 23 \\
2. Plans and Schd. & $-.20(.07)$ & $-.09(.07)$ & $.20(.06)$ & 8 \\
\hline B. Personal mode & $-.33(.06)$ & $.08(.06)$ & $.07(.06)$ & 14 \\
1. Vertical chann. & $.03(.08)$ & $.07(.08)$ & $.13(.07)$ & 2 \\
2. Horizontal chann. & $.51(.07)$ & $.04(.06)$ & $.01(.06)$ & 28 \\
\hline C. Group mode & $.57(.06)$ & $.19(.05)$ & $-.05(.06)$ & 45 \\
1. Sched. meetings & $.48(.06)$ & $.23(.06)$ & $-.03(.05)$ & 39 \\
2. Unsched. meetings & $.60(.06)$ & $.08(.06)$ & $-.07(.06)$ & 43 \\
\hline \hline
\end{tabular}




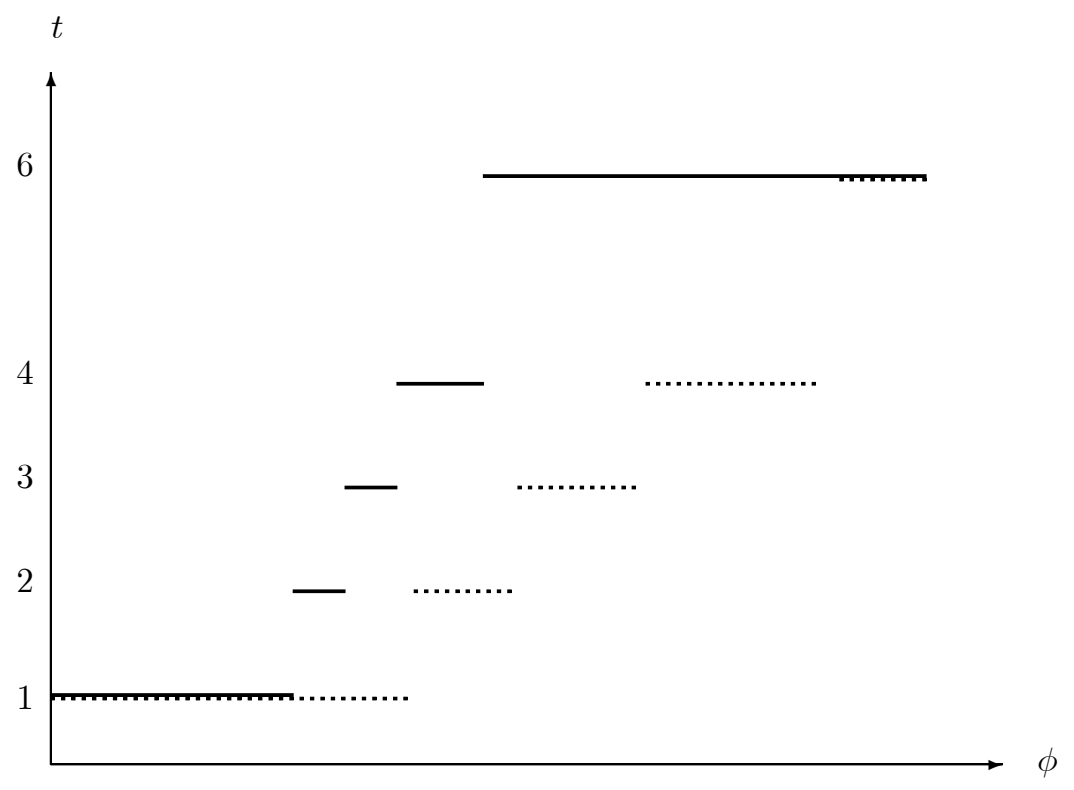

FIGURE I: Example 2. Equilibrium level of the quality of task bundling, $t$, as a function of importance of adaptation, $\phi$. The thick line denotes the case where $p$ is chosen endogenously whereas the discontinous line denotes the case where $p$ is exogenously set. 


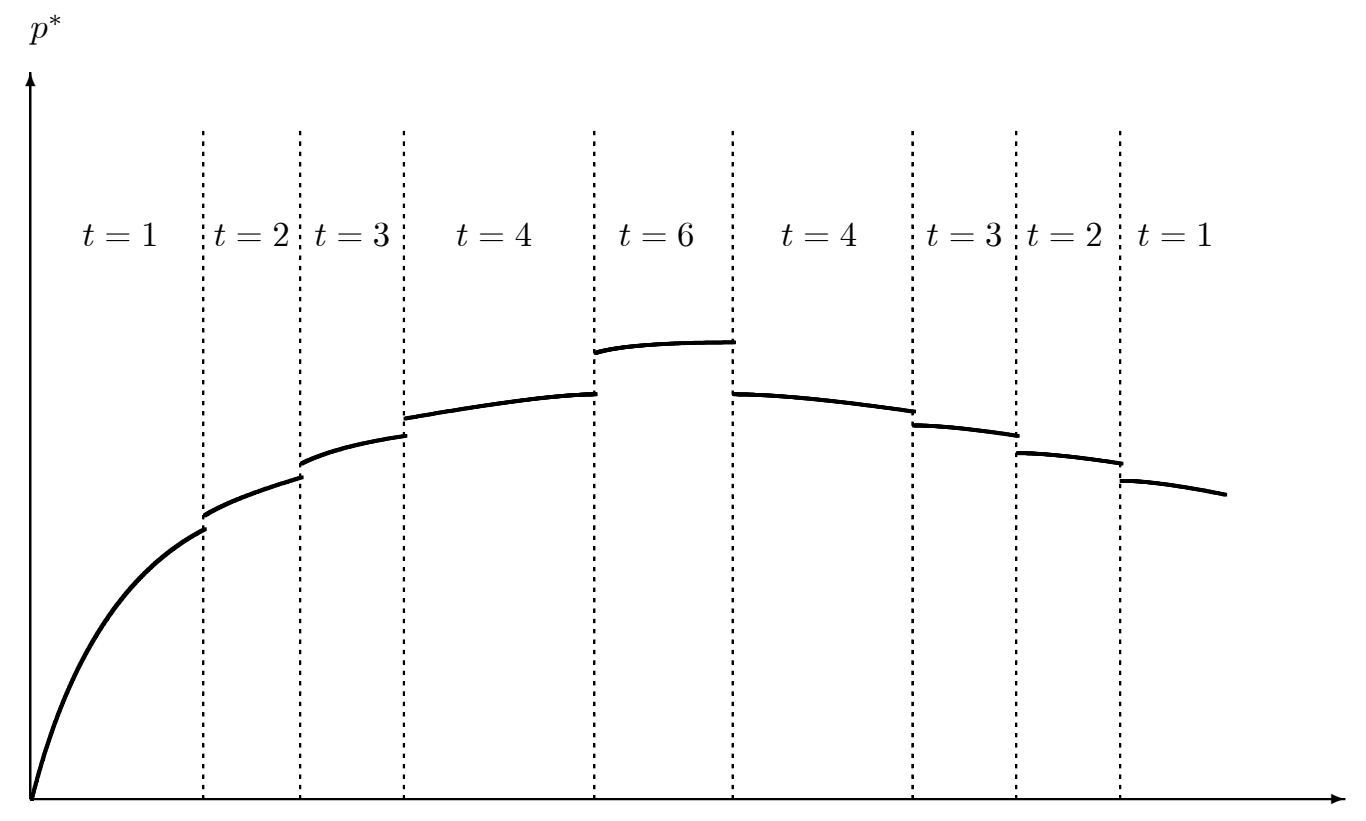

FIGURE II - Panel A: Example 3. Equilibrium level of the quality of horizontal communication, $p$, and task bundling, $t$, as a function of task interdependence, $\beta$.

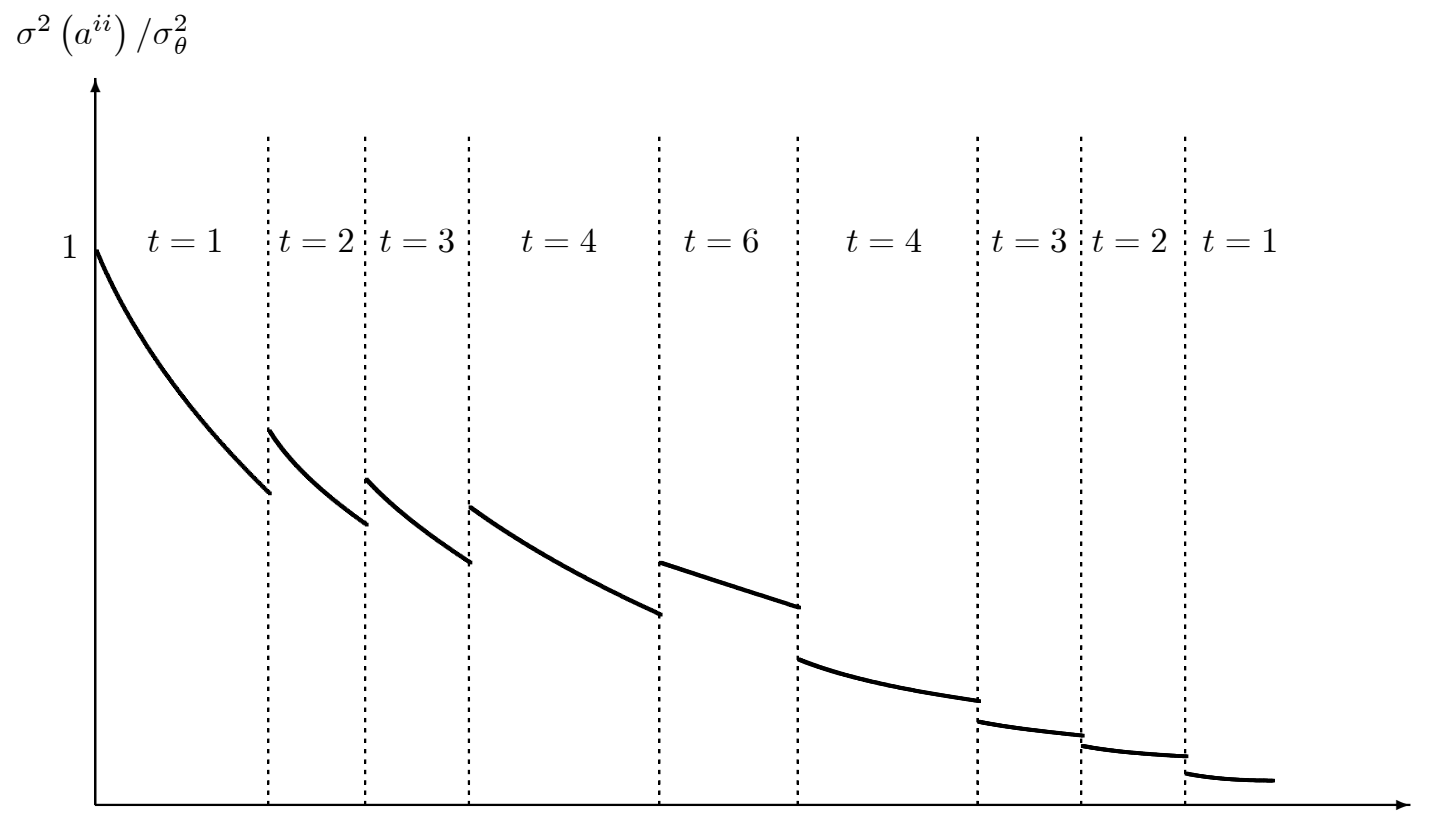

$\beta$

FIGURE II - Panel B: Example 3. Equilibrium level of employee discretion per unit of $\sigma_{\theta}^{2}$, that is, $\sigma^{2}\left(a^{i i}\right) / \sigma_{\theta}^{2}$, as a function of task interdependence, $\beta$. 


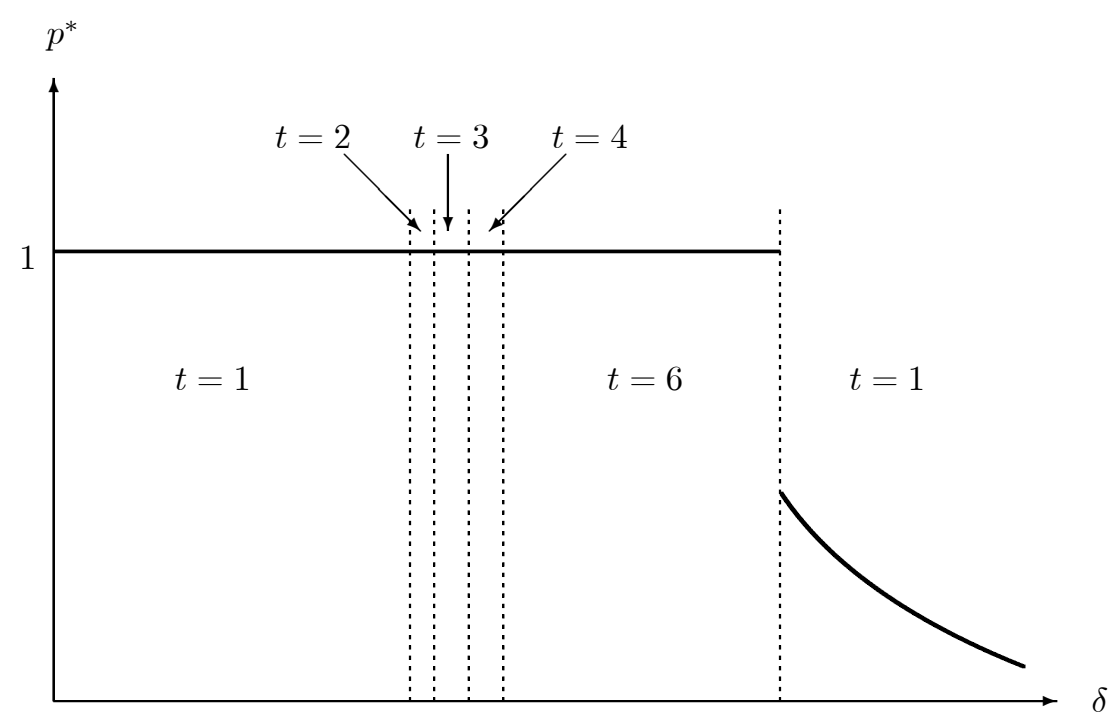

FIGURE III: Example 4. Equilibrium level of the quality of horizontal communication, $p$, and task bundling, $t$, as a function of communication costs, $\delta$. 